\title{
The relationship between men's early maladaptive schemas, rape myth acceptance and self-reported likelihood of using force and raping
}

\begin{abstract}
Global efforts to understand sexual violence against women (SVW) persistently reinforce the magnitude and versatility of this multifaceted problem. Particularly, more thorough comprehension of the structures underlying SVW is warranted. The present study aims to examine the association between early maladaptive schemas (EMSs), rape myths acceptance (RMA) and self-reported proclivity to rape (RP). It was hypothesized that higher RMA is correlated with elevated RP, and that participants will report an increased tendency to use force rather than raping. Moreover, participants with elevated RP are more likely to endorse schemas from the disconnection/rejection domain, and that schema types are unlikely to vary among participants with the tendency to use force rather than raping. In total, 150 male students, who were either citizens or residents in Singapore, from an international university based in Singapore completed a series of self-reported measures assessing RMA, RP, EMSs and social desirability. The findings confirmed that higher RMA predicted increased RP, and participants reported the use of force rather than raping. Although, RMA did predict RP significantly, the predictive power of 4.3 percent was rather low. Elevated RP was predicted by the Unrelenting Standards/Hypercriticalness, Punitiveness and Abandonment/Instability schemas. Furthermore, there were no differences in the schemas endorsed among participants with an increased tendency to rape. The present study has implications for clinical and forensic psychology and further research on SVW in Singapore, primarily due to the plausible influences of culture on the findings. Preliminary support is provided for more thorough research on the use of Schema Therapy in rehabilitating sexually aggressive behaviours.
\end{abstract}

Keywords: early maladaptive schemas, rape myths, self-reported rape proclivity, singaporean males
Volume 6 Issue 6 - 2016

\author{
Sanveen K Kang, Nenna Ndukwe, Daniel \\ Fassnacht \\ Clinical Psychology, James Cook University Singapore, Singapore
}

Correspondence: Sanveen Kang, James Cook University Singapore, Email sanveen.kang@jcu.edu.au

Received: October 21, 2016 | Published: November 18, 2016

\section{Introduction}

Rape of women has persistently been misunderstood. Women are often seen as willing partners rather than preys. ${ }^{1}$ Lifetime prevalence rates of violence against women (VAW) are reaching up to 50 percent in some countries. ${ }^{2}$ Predisposition towards participating in or being a victim of VAW is not differentiated by socio-economic status, professional barrier, caste, age and geographical boundaries. ${ }^{3}$ VAW conceptually varies across cultures in terms of definition and manifestations of the issue. There is no unanimous terminology for describing VAW. ${ }^{4}$ The international consensus for VAW is "any act of gender-based violence that results in or is likely to result in physical, sexual, or psychological harm or suffering to women, including threats of such acts, coercion or arbitrary deprivation of liberty, whether occurring in public or private life". 5

Sexual aggression has been most widely researched form of VAW. ${ }^{6}$ By definition the concept of sexual aggression extends beyond sexual assault, intimate partner violence, harassment, and includes any sexualized behaviour, resulting in the feelings of intimidation and persecution. The diversity of the acts that constitute sexual aggression is reflected in the literature as evidenced by the interchangeable use of various terminologies, such as sexual aggression, coercion, offence, violence and assault. ${ }^{7}$ In fact, thismulti-faceted dimension of sexual aggression contributes to the lack of information about its frequency and consequence. ${ }^{8}$

\section{The prevalence of rape and sexual aggression}

Rape statistics consistently demonstrate the pervasiveness and magnitude of this problem; with the rates being on the rise globally.
The number of convicted rape cases in Singapore has risen by 49.2 percent between January to June 2014 and the same period in 2013 (SPF, 2014). These statistics are not representative of martial rape; as marital rape is not recognized under the Singapore law. It can be assumed that some VAW in Singapore occurs within intimate relationships and, that the actual scope of this issue is unknown..$^{10}$ In the United States, one in five women reported being raped at some point in their lives. ${ }^{11}$ Similar trends have been observed globally. A United Nations survey revealed that one in five women have experienced either a completed or attempted rape in their lifetime. ${ }^{12}$ Up to 58 percent of male respondents acknowledged participating in some form of sexual assault, with 14 percent of them reporting committing a completed rape. Comparable rape perpetration rates have been found in the United Kingdom and Germany. ${ }^{13}$ Despite these alarming consistently high rates, the true prevalence of rape is yet to be understood. Researchers often advise that these rates ought to be interpreted with caution; primarily because of the varied terminologies used to define rape, absence of cross-culturally validated measures of violence and systematic trend of under-reporting. ${ }^{14}$

Current rape statistics may misrepresent the problem's magnitude, as abuse is often not reported to law enforcement. ${ }^{15}$ Unreported cases can be estimated to be significantly greater than those reported. ${ }^{16}$ The AWARE Convention on the Elimination of All Forms of Discrimination against Women ${ }^{17}$ shadow report confirms that Singaporean women under-report sexual violence against themselves. ${ }^{17}$ Statistics may also only reflect the proportion of convicted rape cases; which may merely be a small percentage of those reported. ${ }^{15,18}$ Society appears to be preoccupied with the notion of authentic rape. Real rape is perceived 
as only to happen when the rapist is a stranger, physical force is used, injuries are sustained by the victim and the incident is promptly reported. ${ }^{19,20}$ However, majority of sexual assaults are often committed by acquaintances. Concomitant to enthrallment about the authenticity of the rape are the perceptions of blame and responsibility. Research revealed that victim blame is associated with the provocativeness, character and attractiveness of the victim. ${ }^{21}$ These beliefs (i.e., rape myths) are recognized to be entrenched in society and even appear to be relevant in ascertaining the gravity of an assault within the criminal justice system..$^{22,23}$ As a result, fear of victim blame appears to be central to the underreporting of rape. ${ }^{15}$

\section{Research on rape}

Despite the increase in research on rape, there appears to be limited reliable data. This is particularly in the areas of the true extent of the issue, its repercussions, risk factors and the variation of these across cultures and settings, and the effectiveness of intervention programs. ${ }^{14}$ Much of the data currently available on rape is also obsolete and based on specific populations. ${ }^{24}$ Clinical and social approaches of studying rape have been developed. ${ }^{25,26}$ The current study will be based on the latter category, emphasizing on the naturalistic setting, where participants are university students. Focusing on self-report studies of male students may be disputed to render findings which could be difficult to generalize to other adult male subgroups (i.e., older or less educated). Yet, these studies are deemed relevant as they emphasize the pervasiveness of sexual aggression. ${ }^{27}$ Previous research has demonstrated that convicted rapists are generally unrepresentative of sexually aggressive men within the general population. ${ }^{28}$

\section{Factors associated with sexual aggression and offending}

Men are consistently presented with two clear systems of meeting their sexual needs: either appropriate (i.e., through an intimate consensual relationship) or inappropriately means (i.e., through the use sexual aggression). Genetic factors may predispose one to purse an explicit need (i.e., sexual intercourse), however, environmental factors encourage the approaches used to meet this need..$^{29}$ Genetic influences related to sexual offending include aggressiveness and impulsiveness. ${ }^{30}$ physiological variations in sex drive, neurotransmitter functions and hormone levels, ${ }^{31,32}$ and the consumption of substances. ${ }^{33,34}$ Existing inquiries have also underlined the involvement of social factors in influencing rape statistics, such as viewing pornography $y^{35-38}$ and the legalization of prostitution..$^{39}$ The latter is associated with a reduction of rape incidences as prostitutes often are victims of rape ${ }^{40}$ further complicating our understanding of the extent of the problem. However, this tumultuous relationship between pornography and sexual assault has been shown to be inconsistent. ${ }^{41}$ Environmental stressors in the life of children with a high risk for sexual offending include empathy deficits, poor parental attachment bonds, low self-esteem, poor quality of relationships, deficient emotional coping strategies and cognitive deficits. ${ }^{42,43}$ Sexual offenders often report a history of childhood physical and emotional abuse, parental criminality ${ }^{44}$ and observing inter-parental aggression. ${ }^{45}$ Specifically, rape perpetrators also report cruelty to animals, exposure to violent media and insecure and avoidant attachment bonds. Other factors include adolescence being a developmentally crucial time frame, where the exhibition of antisocial behaviour is viewed as more age-normative; ${ }^{46}$ presence of sexualized coping mechanisms and deviant fantasies; ${ }^{42,47}$ diminished capacity for empathy $;{ }^{48}$ insufficiencies in intimacy skills; $;{ }^{49}$ distorted thinking styles both enable sexual aggression and offending and, serve as post-offence strategies in attempt to maintain self-esteem. ${ }^{50,51}$

\section{Theories related to sexual aggression and offending}

Single factor, neuropsychological and multifactorial theories have been developed to explain sexual aggression. Researchers have repeatedly conveyed the need for a global theory that assimilates all the germane factors into one coherent theoretical structure. ${ }^{52}$ Systematically, researchers have attempted to formulate theories attempting to explain the multiple factors which instigate sexual aggression and have concluded that the multi-causal nature of sexual offending supports theoretical pluralism..$^{53,54}$ Among the multifactorial theories, the Confluence model ${ }^{55}$ is most relevant to the current study as it relates to rape proclivity. Numerous features are expected to converge together to establish sexual aggression. Some generalization between the domain specific factors and the role of the social environment has been accepted by the model. It has been used to distinguish males who show sexual aggression, ${ }^{56}$ and are sexually coercive. $^{37,55}$

Three risk factors for sexual aggression have also been identified: motivation to perpetrate, the presence of an opportunity and diminished internal and external inhibitions. ${ }^{54}$ An individual's probability to sexually aggress against a woman is the result of the interaction between two developmental pathways, the Hostile Masculinity and Sexual Promiscuity/Impersonal Sex pathways. ${ }^{38,55,57}$

The Hostile Masculinity pathway is related to the acceptance of attitudes related to VAW, hostility towards women and narcissistic personality traits. Developmentally, abusive and chaotic family environments may contribute to the progression of unfavourable beliefs about the relationship between a man and woman. Meanwhile, the Sexual Promiscuity/Impersonal Sex pathway is related to early childhood experiences of abuse and conflictual home environments, a history of adolescent delinquency, promiscuity and impersonal sex..$^{58}$

Both pathways tend to converge and interact with each other. ${ }^{55,57}$ The degree to which traits related to the hostile pathway are possessed, directly determines the probability of sexual promiscuity resulting in sexual aggression. Moreover, dysfunction in the development of personality is reflected in both models. The relationship between antisocial behaviour and early sexual behaviour with multiple partners, which is reflective of insecure attachment underpinnings, has been indicated to be an important connection to sexual aggression. ${ }^{59}$ Antisocial personality traits, which are related to the perception of fewer costs of sexual aggression and force, may be imperative to predicting sexually aggressive behaviour. ${ }^{58,59}$ The current study aims at better understanding the relationship between three independent constructs: early maladaptive schemas, rape myths acceptance and self-reported attraction to sexual aggression (i.e., use of force and rape).

\section{Early maladaptive schemas}

Literature on attraction to sexually aggressive behaviours and offending has consistently supported the role of cognitions in the conservation of the phenomenon. For the purpose of this paper, the terms schemas and early maladaptive schemas (EMSs) will be used interchangeably. EMSs develop in early childhood or adolescence, when core emotional needs are unmet, during interpersonal interactions within primary relationships. ${ }^{60}$ They serve as realitybased depictions of the individual's environment. ${ }^{61}$ While not all EMSs are products of maltreatment, they are reliably destructive and caused by deleterious incidents which repeat themselves consistently during childhood and adolescent years. ${ }^{61}$ Schemas can be considered 
our minds' maps to understanding ourselves and the world around us, while determining our emotional and behavioural reactions. EMSs function by distorting information about the self and others through unconscious information processing, resulting in negative automatic thoughts and concomitant emotional experiences. ${ }^{61-63}$ They are highly generalized and resistant to change. ${ }^{64}$ Young et al., ${ }^{61}$ categorized 18 EMSs into five broad domains. Since the number of EMSs is not finite, the focus ought to be on the five broad domains. ${ }^{62}$ Henceforth, the current study will endeavour to focus on schema domains, instead of only the individual schema types. These include: disconnection and rejection; impaired autonomy and performance; impaired limits; other-directedness; over-vigilance and inhibition domains.

Individuals with schemas in the disconnection and rejection domain are incapable of establishing secure attachment and fulfilling interpersonal relationships, due to beliefs that their needs for stability, safety and love are unlikely to be met. Families of origin are usually unstable (Abandonment/Instability), abusive (Mistrust/Abuse), cold (Emotional Deprivation), rejecting (Defectiveness/Shame) or isolated from the rest of the world. ${ }^{61}$

The impaired autonomy and performance domain is related with the incapacity to separate from parental figures and operate autonomously. Families of origins comprise of either overprotective or contrastingly dismissive parents. These individuals are disposed to feeling unequipped to manage everyday responsibilities without support (Dependence/Incompetence), immense fear due to beliefs of being unprepared to cope with impending catastrophe (Vulnerability to Harm or Illness), believing that one will fail due to general inadequacy (Failure), and feeling fused with others owing to the absence of a clear sense of identity and direction (Enmeshment/Undeveloped Self). ${ }^{61}$

Meanwhile, the impaired limits domain is associated with insufficiently developed internal limits. Families of origin may be permissive or overtly indulgent. These individuals are likely to assume superiority to others and feel entitled to special rights (Entitlement/ Grandiosity), and have challenges with regulating emotions and impulses resulting in insufficient self-control and frustration tolerance with attempting to achieve their personal goals (Insufficient SelfControl/Self-Discipline).$^{61}$ Individuals with the other-directedness domain value pleasing and meeting the needs of others, even if it is at the expense of their own needs. Families of origin are based on conditional approval, and during their earlier years the freedom to follow their natural inclinations was not developed. As adults, these individuals may surrender control to others when they feel coerced, primarily to avoid unpleasant emotions such as anger, retaliation or abandonment (Subjugation); attempt to meet the needs of others at the expense of their own in order to gain self-esteem, maintain emotional connectivity with someone who is viewed as needy or to spare others the experience of pain or guilt (Self-Sacrifice); and attainment of approval or recognition from others with the rationale to develop a secure sense of self (Approval Seeking/Recognition Seeking). ${ }^{61}$ Lastly, the overvigilance and inhibition domain is associated with the drive to suppress unprompted and natural feelings or impulses. The primary intention is to appease inflexible internalized rules about one's own performance, often at the detriment of positive emotions and experiences. The family of origin is strict; where self-denial and self-control dominate spontaneity and pleasure. Often times, there appears to be a pervasive focus on negative life events, in combination with the obsession that things will eventually go wrong (Negativity/Pessimism); inhibition of natural emotions, behaviours and communication due to the fear of being criticized or losing control over impulses, and hence seemingly presenting as being cold and withdrawn (Emotional Inhibition); belief that disapproval or shame can be avoid through the achievement of personally set high standards, which inevitably produce feelings of pressure and hyper criticalness (Unrelenting Standard); and opinion that harsh punishment ought to follow mistakes, causing anger and intolerance of others and even self during instances where these selfimposed standards are unmet (Punitiveness). ${ }^{61}$

Cognitive schemas and distortions are acclaimed focal targets in sex offender treatment. ${ }^{65}$ Cognitive distortions and underlying core structures, such as EMSs, have been deployed to distinguish among the varieties of sex offenders. ${ }^{51,65}$ However, research on the role of EMSs in the tendency to engage in or the self-reported attraction towards rape and the use of force to obtain sex, is scant. Instead, most of research focuses on offence-specific cognitive distortions. ${ }^{51}$ While EMSs are not specific to offenders' impressions about their victims or perception of their crimes, they offer a wealth of information related to both the self-perception and world view of sex offenders, and consequentially reflecting their level of psychological and social adjustment. ${ }^{65}$ Unlike surface level cognitions, EMSs are not restricted to the offender's explanations about the victim's intentions or the events leading to the crime, which serve as post hoc justifications for the crime. ${ }^{66,67}$

Cognitive distortions have been shown to influence sexual recidivism. ${ }^{68}$ It can be anticipated that since EMSs drive cognitive distortions, they will be present in rape perpetrators and men within the general population who report proclivity to sexual aggression. Hence, EMSs may undoubtedly be crucial to matters related to sexual perpetration and recidivism. However, the limitation of available literature is the variability in findings. Knowledge derived from these studies may be culturally specific, and may not generalize to the Singaporean population. According to the Schema approach, cultural influences are integral in EMSs development. This inconsistency warrants further investigation. Rape perpetrators in the United States endorsed higher levels of Instability/Abandonment and Defectiveness/ Shame schemas, and only somewhat higher levels of the Emotional Deprivation schema in comparison to non-perpetrators. Endorsements of varied EMSs were associated with predicting perpetration status. ${ }^{69}$ Moreover, 74 percent of sexually abusive British adolescents presented with clinically significant EMSs.

Emotional inhibition, social isolation/alienation and mistrust/ abuse were identified as treatment targets for the clinical sample. ${ }^{44}$ An Iranian study reported that elevated scores on the disconnection/ rejection, and impaired autonomy/performance schema domains may be related to the propensity towards sexual assault and rape. ${ }^{70}$ Furthermore, Sigre et al., ${ }^{71}$ found that male college students from Northern Portugal who reported sexual coercion of women endorsed higher levels of the Mistrust/ Abuse, Dependence/ Incompetence and Negativity/ Pessimism schemas. Some of the factors related to sexual offending previously described are known to be linked to general criminal behaviour or antisocial orientation. ${ }^{53,72}$ While no diagnostic criterion is specific to sexual offenders as a unitary group, ${ }^{73,74}$ studies have determined a relationship between antisocial orientation and criminal recidivism; including sexual offending. ${ }^{75}$ Despite the established relationship between psychopathology and sexual offending, and EMSs and psychopathological symptoms, there is limited research on the role of EMSs in the propensity towards sexual aggression. ${ }^{76}$

\section{Rape myths}

Theories attempting to explain rape occurrences often emphasize on rape myths, ${ }^{77,78}$ which are prescriptive beliefs that operate to 
downplay or justify sexual violence against women. ${ }^{28}$ Effectively, they are seen as cognitive schemas. ${ }^{79}$ Rape myths manage the elucidation of pertinent information from rape cases and contribute to rape prevalence. ${ }^{28,79,80}$ Burt $^{78}$ expounded a causal model of rape myth acceptance (RMA). The acceptance of interpersonal violence appears to be the strongest predictor of RMA. Inherently, RMA is related to sex-role stereotypes, sexual conservatism, adverse sexual beliefs ${ }^{78}$ and the use of sexual coercion within the community. ${ }^{81}$ RMA is rampant in Western, ${ }^{82,83}$ South African ${ }^{84}$ and Asian societies. ${ }^{85}$ While rape myths are pervasive, their influence appears to vary based on gender, culture and time periods. ${ }^{83}$ These beliefs are firmly held primarily because they serve psychological motivations. ${ }^{86}$ For that reason, RMA appear to serve several functions. Among these functions is a general cognitive schema and prediction of proclivity to rape. ${ }^{2}$ Rape myths tend to influence the interpretation of information which is case specific. They allow rape perpetrators to disable social norms against hurting others and reduce the anticipated negative consequences. ${ }^{2}$

Forthrightly, rape myths corroborate support for victim blame and may lead to unrecognized rape. Rape myths have been illustrated to be a component of a learned system where consensual sex is related with power. Sexually aggressive men consciously make the decision to persist with advances despite a realization that their advances are unsolicited ${ }^{87}$ Individuals who hold a higher level of rape myths also have a lower probability of recognizing and labelling situations as encompassing a rape.$^{83}$ Astonishingly, beliefs about women and sexual aggression have been shown to be comparable for rape perpetrators and non- perpetrators, and are not always distinct between the two groups. In fact, the variability is based on the degree as opposed to the nature of the beliefs. ${ }^{88}$

\section{Attraction to sexual aggression}

Attraction to sexual aggression is the belief that sexual aggression is likely to be sexually arousing for both the perpetrator and victim, and is related to a male's propensity towards aggressing in the absence of the fear of punishment or other inhibitory factors. Sexual aggression has been suggested to be attractive for men from the general population at varying degrees..$^{89}$ Men attracted towards sexual aggression are more likely to endorse rape myths and endorse that rape is acceptable under specific situations. ${ }^{90}$ Proclivity to rape,defined asmen's self-reported probability to rape under hypothetical conditions whereassurance of avoiding identification and punishment is provided, is recognized as being crucial in understanding the perpetration of sexual aggression. Perception of rape, sexual arousal to violence and aggressive behaviour may explain rape proclivity. ${ }^{50}$ The relationship between rape proclivity and aggression towards women was positively proportional. ${ }^{38,91}$ Individuals who endorse higher RMA reportedly have a higher proclivity to rape or commit sexual violence. ${ }^{28,92,93}$ In fact, the causal relationship between RMA and proclivity to rape is well-established ${ }^{28}$ through European and North American research, which demonstrated that a considerable percentage of males report some proclivity towards sexual aggression. ${ }^{28,50,92}$ Patterns of attitude towards rape held by men in the general population and rape perpetrators were discovered to be similar. ${ }^{94}$ Furthermore, proclivity to rape has been shown to be influenced by social expectations regarding rape myths. Male students who received feedback regarding their peers' elevated endorsement of RMA reported that they were more likely to commit sexual violence. The converse was revealed for those who received feedback regarding their peers' lower endorsement of RMA..$^{5}$ Social desirability may, therefore, be an influencing factor when considering self-disclosure about these two constructs. It reflects the desire to present oneself in a manner coherent with accepted social norms, rather than reflecting one's personal beliefs. ${ }^{96}$ Given the substantial support for the association between RMA and rape proclivity, and that RMA are generally accepted within the general population, understanding their prevalence within society and taking efforts to challenge them at a societal level maybe key in minimizing the proclivity to rape of males within the general population. Rape proclivity may hold the key to distinguishing individuals at risk for engaging in sexual aggression from those who are not a risk.

\section{Current study}

The current project is an extension of the study by Bohner et al., ${ }^{28}$ Two experiments to explore the pathways underlying the correlation between RMA and proclivity to rape were conducted. In both experiments, the relative cognitive accessibility of rape myths and proclivity to rape was experimentally manipulated through the sequence of completion of the questionnaires which assessed these constructs. Explicitly, this necessitated the completion of the RMA measure prior to that for rape proclivity. The activation of rape myth consistently had a casual influence on the proclivity to rape. Furthermore, elevated levels of RMA towards women were related to higher proclivity to rape. The main limitation of the study was that developmental factors contributing to the endorsement of higher levels of rape proclivity were not assessed. These will be explored in the form of EMSs in the current research. Bohner et al., ${ }^{28}$ also did not account for the variability in the endorsement of the likelihood to use force or rape items. Men from the general population have been shown to be more likely to endorse the use of force as opposed to rape. The current study will assess both the self-reported likelihood to use force and rape. Furthermore, increasing the accessibility of rape myths may heighten awareness of them and increase behavioural inclinations. However, there is insufficient evidence that this relationship is causal. This emphasizes a methodical error in Bohner et al., ${ }^{28}$ study as a longitudinal experimental design was not used to confirm the nature of the relationship. Lastly, another limitation was that social desirability was only accounted for in the second experiment. It may be possible that the smaller effect in first experiment may be somewhat attributable to social desirability. Despite the increasing rate of reported rape cases, sexual aggression is not avidly researched in Singapore or even within the Asian context. Previous research conducted in Asia was typically limited to victimization surveys, ${ }^{97}$ attitudes supporting rape $^{98}$ and to a limited extent, sexual aggression . ${ }^{56,98,99}$ Lim et al., ${ }^{56}$ confirmed that factors influencing sexually aggressive behaviours as per the confluence model ${ }^{55}$ are relevant to Singapore. Evidence for cross-cultural variability in sexually coercive behaviours in Singapore was also corroborated. Cultural views pertaining to sexual matters being taboo have been attributed to the lowered interest in studying sexual aggression in Asia. ${ }^{56}$ Hence, much of the available knowledge in this area comes from research in the West or other nations, which may differ significantly from Singapore. Asian societies have a more collective worldview as opposed to Western cultures. Collectivism promotes a strong sense of filial piety, where parental obedience is highly valued and instilled through firm disciplinarily strategies; ${ }^{100,101}$ which are shunned and considered abusive in Western societies. ${ }^{102-104}$ Therefore, it is likely that developmental factors and childhood experiences associated with sexual violence in the West may differ in the Asian context. With specific regard to Singapore, these dynamics may be even more variable given that its society is a melting pot of Western and Asian cultures. Although the relationship between the RMA, RP and sexual violence is well established, few studies have endeavoured to understand the deep-seated constructs underlying these systems. Research consistently demonstrated that maladaptive beliefs and cognitions are imperative in understanding 
the causation of sexual aggression. ${ }^{105}$ While previous studies have concentrated on offence-specific cognitive distortions, the assessment of underlying cognitive structures and processes has been found to be more meaningful. Cognitive structures provide clarification on the development of the cognitive distortions, and their association with sexual offending. ${ }^{105,106}$ Addressing schemas during treatment maybe more relevant than surface level or specific to situations cognitive distortions.

Dysfunctional schemas have been indicated to make sexual offending more likely as they are known to bias information processing. ${ }^{107}$ Consequently, tumultuous early childhood experiences may suggest that these individuals may endorse EMSs and will have greater attraction towards sexual aggression and are likely to endorse RMA to justify their stance. Interestingly, while research has clearly established that EMSs play a crucial role in the development and maintenance of psychological disorders, interpersonal problems ${ }^{108}$ and other offending behaviour (i.e., murder and drug trafficking;), ${ }^{109}$ little research has been conducted to understand their role in attraction to sexual aggression and the acceptance of rape myths. Hence, the current research aims at identifying specific EMSs which are related to endorsement of RMA and attraction to sexual aggression, particularly the self-reported likelihood to rape and use force. Through the identification of these EMSs, the current study may shed some light on the developmental and environmental factors related to the establishment and maintenance of sexual aggression in men.

Additionally, current research may provide more evidence for the use of schema-based therapy for the treatment for rape perpetrators. Currently, the treatment of choice is multi-faceted group cognitive behaviour programmes which emphasize "offence-specific issues," with treatment targets being social skills training and anger management. Little attention is paid to individual personality traits and dysfunctional beliefs or maladaptive schemas. In the event that maladaptive schemas are found to be present among men in the general population who report proclivity to rape, then the treatment target ought to be the maladaptive schemas, which will be addressed in the format of individual schema therapy as opposed to the groupbased treatments. ${ }^{110}$

\section{Aims and hypotheses}

The current research aims at understanding the following:

a. Is the endorsement of RMAs correlated with higher proclivity to rape women?

b. Are males more prone towards acknowledging the likelihood to rape or use of force?

c. Which EMSs domains are endorsed by males with an elevated self-report proclivity to rape?

d. Do the EMSs vary among men who report an increased likelihood to use force as opposed to rape?

The following hypotheses will be studied in the current research:

\section{Primary Hypotheses}

a. The primary hypothesis will test the relationship between RMA and the proclivity to rape women in a Singaporean sample. It is anticipated that participants who endorsed higher levels of RMA will report higher proclivity to rape.

b. It is anticipated that the participants will report an increased tendency to use force rather than raping.

\section{Secondary Hypotheses}

a. The second hypotheses are more exploratory in nature. Since childhood physical abuse, emotional abuse, ${ }^{111,112}$ parental criminality ${ }^{44}$ and observing inter-parental aggression have been identified as risk factors for sexual offending, it is deduced that the disconnection/rejection domain may best describe the family of origin and developmental experiences of rape perpetrators. Hence, it is hypothesized that participants with elevated levels of proclivity to rape will primarily endorse this schema domain.

b. Previous findings reveal that more men indicated the likelihood to endorse items that use the term "force" rather than "rape". ${ }^{50,89,113}$ It is hypothesized that the schemas endorsed by participants who report likelihood to use force is not expected to differ from those who report likelihood to rape in terms of the types of schema domains endorsed.

\section{Materials and methods}

\section{Design}

Due to scarcity in research on sexual violence against women in Singapore and also that exploring the relationship between Young's Early Maladaptive Schemas, ${ }^{61}$ RMA and self-reported proclivity to rape and use force, there is an absence of underlying theory explaining the relationship between these three constructs. As such, the current study adopted an exploratory approach to further investigate and understand the relationship between the three constructs which appear to be inter-related. Data was gathered from a variety of questionnaires.

\section{Participants and Procedures}

The target group for the current study was male undergraduate and graduate English-speaking students at James Cook University Singapore (JCUS). Participants were recruited through advertisement on research bulletins at the JCUS campus and, a cloud-based research and participant management program. Eligible participants were awarded research credits towards their course requirement to participate in Psychology research, as an incentive for their participation in the study. The inclusion criteria for the study included only participants who were either citizens or permanent residents of Singapore. Participants of homosexual orientation and prior criminal history were excluded from the study. In total, 152 males were recruited for the purpose of this study. However, two participants were excluded as they reported being of homosexual orientation. Participants ranged in age from 18 to 57 years, with an average age of 23.4 years $(S D=4.1)$.Preceding analogue investigations have been conducted with comparable populations, ${ }^{28,114,115}$ as males between the ages 16 and 29years have been revealed to have the highest propensity towards participating in sexually coercive behaviours. ${ }^{116}$

The participants were asked to complete a series of questionnaires assessing male sexual attitudes towards different aspects of sexuality. They were requested to complete all questions in order in which they were presented. Each set of questionnaires include a series of measures in the following prescribed order: Young Schema Questionnaire - Short Form, Third version (YSQ-S3), ${ }^{117}$ Acceptance of Modern Myths about Sexual Aggression (AMMSA), ${ }^{2}$ Revised Attraction to Sexual Aggression Scale (ASA-R), ${ }^{113}$ and MarloweCrowne Social Desirability Scale (MCSDS) ${ }^{96}$ The measure of RMA was always presented before the measure of proclivity to rape and use force since it has been demonstrated through previous research that the activation of rape myths rendered higher endorsements of behavioural inclinations of rape. ${ }^{28}$ 


\section{Measures}

Young schema questionnaire - short form, third version (YSQ-S3): ${ }^{117}$ This is a 90 -item self-report measure which has been shown to effectively identify 18 Early Maladaptive Schemas (EMSs) described in Schema Therapy. ${ }^{61}$ Each EMS is evaluated through five separate items. Participants were requested to rate their degree of agreeableness with each of the items on a 6-point Likert scale, ranging from completely untrue of me (1) to describes me perfectly (6). In conformity with the recommended scoring procedure and previous research, ${ }^{118,119}$ the scores for each schema were summed and then divided by the number of items. This eventually produces a mean score for each schema, which ranges from 1 to 6 . While limited research has been conducted on the YSQ-S3, extensive research has been done on early versions of the YSQ. ${ }^{120}$ The measure has been shown to have high coefficients for test-retest reliability, internal consistency and convergent validity. ${ }^{121,122}$

Acceptance of modern myths about sexual aggression (AMMSA): ${ }^{2}$ This is a 30 -item self-report scale which evaluates understated modern myths about sexual aggression towards women. The AMMSA comprises of five categories of myths:

a. denial of the scope of the problem,

b. antagonism towards victims' demands,

c. lack of support for policies designed to help alleviate the effects of sexual violence,

d. beliefs that male coercion forms a natural part of sexual relationships and

e. beliefs that exonerate male perpetrators by blaming the victim or the circumstances. ${ }^{2}$ Participants were asked to rate their level of agreeableness for each item on a 7-point Likert Scale, ranging from totally disagree (1) to totally agree (7). In accordance with the recommended scoring procedure, all items are positively scored. Total score results from computing the arithmetic mean of item scores across all 30 items, and thus the score can range from 1 to 7 . Higher scores indicate a greater acceptance of modern myths about sexual aggression. ${ }^{2}$ The English version of the AMMSA has been shown to have high coefficients for internal consistency and test-retest reliability. ${ }^{2}$ Moreover, the internal consistency reliability analysis based on the present participant pool revealed that the Cronbach's alpha coefficient for the AMMSA was consistently high at .90 .

Marlowe-Crowne social desirability scale (MCSDS): ${ }^{96}$ This is a 33 -item self-report measure, which has been shown to be an efficient measure of social desirability (see Appendix B for a copy of the MCSDS). Participants were asked to make a forced choice, through a true-false format, when responding to the questionnaire. The MCSDS has two factors: Attribution and Denial. The Attribution subscale consists of 18 items and focuses on the participants' likelihood to sanction socially approved behaviours which are uncommon, such as going out of one's way to help another person who is in trouble. The Denial subscale comprises of 15 items and focuses on the propensity to deny socially disapproved behaviours which are common, such as being jealous of the good fortune of others. Based on the scoring criteria, one point is awarded if True is marked for items in the Attribution subscale and False is marked for items in the Denial subscale. The MCSDS yield a score ranging from 0 (low) to 33 (high social desirability). Higher scores reflect a greater degree of socially desirable responding. Crowne et al. ${ }^{96}$ reported that the instrument's Cronbach's alpha coefficient is .88.Conversely, the internal consistency reliability based on the present participant pool revealed that the Cronbach's alpha coefficient as poor for the Attribution subscale $(\alpha=.58)$, Denial subscale $(\alpha=.68)$ and the total score $(\alpha=.64)$.

Revised attraction to sexual aggression scale (ASA-R): ${ }^{113}$ This is a 9-item self-report measure, which is designed to assess attraction to sexual aggression andself-reported likelihood of committing rape and using force. Participants were asked to rate their actual behaviour and attraction towards a variety of sexual and non-sexual behaviours, on a Likert-scale. Of specific interest to the current study are the likelihood to rape (LR) and likelihood to use force (LF) items on the ASA-R. For both items, participants are asked to rate their likelihood to attempt rape or to use force to obtain sex from an unwilling partner in the event that the participant was sure that no one would find out and he will never be punished for it. Both items are measured on a 7-point Likert scale, ranging from (1) very unlikely to (7) very likely. Moreover, the LR and LF scores can be combined to generate the likelihood to use force and rape score (LRF). The LRF score is an indicator of rape proclivity.

Scores for the LR and LF scales range from 1 to 7, with higher scores indicating a greater likelihood. Meanwhile, the LRF score may range from 2 to 14, with higher scores indicating a greater likelihood. The original and revised ASA has a high internal consistency and validity. According to Voller et al., (2009), the Cronbach's alpha coefficient for this measure is .94. Moreover, the internal consistency reliability analysis based on the present participant pool revealed that the Cronbach's alpha coefficient for the ASA-R was acceptable at .71.

\section{Procedures}

Data collection only commenced after ethics approval was obtained from the James Cook University Australia Human Research Ethics Committee. Detailed information pertaining to the nature of the study, as stipulated by the Ethics Committee, was provided to all interested participants during the recruitment stage. An Information Sheet was provided to each participant following which informed consent was obtained. After completion of the study, a brief summary detailing the objectives of the study and the contact details of agencies in Singapore, who were able to provide on-going support, was provided to all participants. Participants who appeared to be distressed were individually debriefed, assessed for risk for harm to self or others and, referred to external agencies in Singapore, who specialized in working with issues related to sexual aggression.

\section{Statistical analysis}

The statistical software package SPSS, version 21.0 was utilized to conduct all statistical analyses. Unless otherwise stated, an alpha level of .05 was used for all the tests. The primary hypotheses were tested using simple linear regression and paired samples t-test. Subsequently, the secondary hypotheses were explored using stepwise multiple regression and independent samples t-test. The effects of social desirability were also considered in the above mentioned analyses.

\section{Results}

\section{Relationship between RMA and proclivity to rape}

Since it was predicted that participants who endorsed higher levels of RMA will report higher proclivity to rape, a simple linear regression was performed to investigate this relationship. Screening of the data for violations of assumptions revealed that the assumptions for:

$$
\text { a. } N: k \text { ratio, }
$$



b. outliers and
c. multicollinearity were not violated.

As the assumption for normality was violated, both variables (i.e., RMA and LRF) were transformed to a best fit model with a power function (i.e., natural logarithm), in an attempt to ascertain if normality could be improved in the linear regression. The normality assumption was not met, albeit improving after the transformation. The analysis revealed that RMA scores significantly predicted self-reported proclivity to rape $(\beta=.209, t(148)=2.59, p<.01)$.Participants who endorsed higher levels of RMA reported a higher proclivity to rape, when the effects of social desirability were not controlled ${ }^{1}$.The results of this analysis revealed that the percentage of accountability of this model was $R^{2}=.043$, suggesting that the predictive power of the model was 4.3 percent.Overall, the first hypothesis was confirmed.

\section{Propensity to report the use of force or rape}

It was anticipated that the participants would report an increased tendency to use force rather than raping. The analysis revealed that higher scores were reported for the likelihood to use force $(M=$ 2.37; $S D=1.67)$ as compared to the likelihood to rape women $(M=$ $1.67 ; S D=1.37$ ), when the respondents were assured that their identity would remain confidential and they could avoid punishment. Furthermore, 26.67 percent of participants provided a response of 2 and above on the item assessing the likelihood to rape women, as compared to 51.33 percent of participants on the item related to the likelihood to use force. In order to further examine the difference between these two related variables, a paired samples t-test was carried out. Screening of the data for violations of assumptions revealed that the assumption for the scale of measurement was met and there was no missing data. The assumptions for normality and normality of difference scores were not met. In some cases, the difference in scores takes the value 0 (i.e., when the scores for the two variables were the same). It is arithmetically not possible to take a natural log of zero. As such, no data transformation was considered due to the nature of the present study. Moreover, it has been found that only a minority of the data collected in educational and psychological research follows univariate normal distributions. ${ }^{123}$ The difference in the propensity to report the use of force in comparison to rape was statistically significant, $t(149)=-5.93, p<.001$, Cohen's $d=0.48$. Henceforth, the current data provides support for this hypothesis and corroborate previous findings revealing that men are more likely to endorse items that use the term "force" instead of "rape". $55,89,113$

\section{Relationship between elevated proclivity to rape and schemas}

It was hypothesized that participants with elevated levels of proclivity to rape will primarily endorse schemas from the disconnection/rejection domain. The order of entering predictors into a regression model is typically determined by theory. ${ }^{124}$ Due to the explanatory nature of the secondary hypothesis and the absence of theory explaining the development of early maladaptive schemas in males with an elevated proclivity to rape, a stepwise multiple regression was carried out. A stepwise multiple regression is performed when the aim is to identify the best combination of independent variables (i.e., schemas) that will predict the dependent variable (i.e., elevated proclivity to rape). The assumption of the absence of significant correlation between each variable-pair was met. Given that LRF scores may range from 2 to 14, for the purpose of the current analyses, a score of seven and above on the LRF was categorized as an elevated proclivity to rape. The mean scores of each of the 18 EMSs from the YSQ-S3 were entered into the model.
The prediction model contained three out of the 18 predictors. ${ }^{2}$ These three predictors were the Unrelenting Standards/Hypercriticalness, Punitiveness and Abandonment/Instability schemas. The model was statistically significant, $F(3,146)=6.819, p<.001$, and accounted for 12.3percent of the variance of proclivity to rape $\left(R^{2}\right.$ $=.123$, Adjusted $R^{2}=.105$ ). Elevated levels of proclivity to rape were primarily predicted by higher scores on the Unrelenting Standards/ Hyper-criticalness schema, lower scores on the Punitiveness schema and to a lesser extent lower scores on the Abandonment/ Instability schema. With the sizeable correlations between the predictors, the unique variance explained by each of the variables indexed by the squared semipartial correlations, was relatively high Unrelenting Standards/Hyper-criticalness, Punitiveness and Abandonment/ Instability schemas uniquely accounted for 24.8percent, 26.7percent and 18.1percent of the variance of proclivity to rape. Table 1 demonstrates the unstandardized $(B)$ and standardized $(\beta)$ regression coefficients, squared semi-partial (or 'part') correlations $\left(s r^{2}\right)$ and significance $(p)$ for each predictor in the regression model.

\section{Variability in schema types among men with propensity to report the use of force or rape}

The preceding analysis revealed that men are more likely to endorse items that use the term "force" instead of "rape." As such, it is hypothesized that the schemas endorsed by participants who report likelihood to use force will not differ from those who report likelihood to rape. To determine if there was a statistically significant difference among these two groups, an independent samples t-test was conducted for each of the three identified schema types.

Unrelenting standards/Hyper-criticalness schema: Preliminary analysis revealed that participants who reported an increased likelihood to use force $(M=3.92, S D=.72)$ endorsed higher scores on the Unrelenting Standards/Hyper-criticalness schema, as opposed to those who reported an increased likelihood to rape $(M=3.71, S D=$ .93). The Levene's test for equality of variance did not reach statistical significance $(F=2.34, p=.131)$, suggesting that the variability in the two conditions is not significantly different. The difference in the means was not statistically significant, $t(66)=.92, p=.362$, twotailed. In summary, for the Unrelenting Standards/Hyper-criticalness schema, there is no difference between men who endorsed the increased likelihood to rape as opposed to the use of force.

Punitiveness schema: Preliminary analysis revealed that participants who reported an increased likelihood to use force $(M=3.31, S D=$ $.95)$ endorsed slightly higher scores on the Punitiveness schema, as opposed to those who reported an increased likelihood to rape $(M=$ $3.15, S D=1.10)$. The Levene's test for equality of variance did not reach statistical significance $(F=1.97, p=.166)$, suggesting that the variability in the two conditions is not significantly different. The difference in the means was not statistically significant, $t(66)=$ $.53, p=.600$, two-tailed. In summary, for the Punitiveness schema, there is no difference between men who have elevated self-reported likelihood to rape and men who endorse the use of force.

Abandonment/Instability schema: Preliminary analysis revealed that participants who reported an increased likelihood to use force $(M=2.63, S D=.95)$ endorsed lower scores on the Abandonment/ Instability schema, as opposed to those who reported an increased likelihood to rape $(M=3.17, S D=1.43)$. The Levene's test for equality of variance is statistical significance ( $F=10.13, p=.002)$, suggesting that the variability in the two conditions is significantly different. The t-test was not statistical significant, $t(14.57)=-1.29, p=.216$, twotailed, suggesting that the difference in the means was not statistically significant. In summary, for the Abandonment/Instability schema, 
there is no difference between men who endorsed the increased likelihood to rape as opposed to the use of force.

In summary, the hypothesis that participants who report likelihood to use force will not differ from those who report likelihood to rape was supported for all of the three predicted EMSs.

A hierarchical multiple regression analysis was also carried out to account for the influence of social desirability in the relationship between RMA and proclivity to rape. However, since social desirability was not significantly predicting proclivity to rape, this analysis was not included in the reported analysis. ${ }^{2}$

Since social desirability was not significantly predicting proclivity to rape, only analyses that did not control for the effects of social desirability are included in the reported analysis.

\section{Discussion}

Violence towards women has been inflicted by men throughout recorded history and across cultures. ${ }^{125}$ Society has recognized that sexual aggression towards women is a colossal problem. Sexual violence not only poses a social threat, but also violates human rights and jeopardizes global economy. ${ }^{126}$ Rape appears to be the most feared form of violence against women, ${ }^{21}$ and has been occurring at an alarmingly increasing frequency. ${ }^{2,127}$ To further contribute to the complexity of this problem, the rates of rape and even unreported cases is expectantly increasing globally. ${ }^{15,128}$ Investigations into rape are versatile and have continuously aimed at expanding available knowledge about the perpetrator, victim and the available treatment options for both of these parties. The plight in the development of an etiological theory that effectively explains sexual aggression has not only been ongoing and daunting, but continuously illuminating the need for an integrated theory. While significant gains in research have been made over the years, little has been done to bridge the gap between the existing and overlapping knowledge available from the existing theories. As such, several areas pertinent to understanding sexual aggression towards women remain to be unexplored. Of particular relevance to the current study, these unexplored territories include understanding the pertinent underlying structures which may encourage the acceptance of the rape "culture" and increase one's appeal towards sexual violence. It has been established that rape is a learnt behaviour ${ }^{81}$ and a majority of rapists are recidivists. ${ }^{128}$ Previous research suggests that it is a phenomenon that can be influenced and may be responsive to intervention. In fact, addressing schemas during treatment has been recognized as being more relevant than addressing cognitive distortions, which are surface level or specific to situations. ${ }^{105}$ This corroborates further support for understanding the environments and unique belief systems and/or thought processes which increase the propensity of men to engage in such an abhorrent act.

The current study was an extension of Bohner et al.,${ }^{28}$ study on the pathways underlying the correlation between rape myth acceptance (RMA) and proclivity to rape. There were two experiments in their study. In both of them, the cognitive accessibility of rape myths and proclivity to rape was experimentally manipulated through the sequence the questionnaires were completed. This correlational relationship between RMA and proclivity to rape was attributed as being due to an underlying causational pathway. ${ }^{28,129}$ While increasing the accessibility of rape myths may heighten the awareness of their existence and increase behavioural inclinations, there is limited evidence that this is in fact a general causal relationship. Such a conclusion seems to emphasize a methodical error as a longitudinal experimental design was not used to confirm the nature of the relationship. As such, the present study aimed at determining whether this established correlational relationship held as being true in Singapore. Analogous to Bohner et al. (1998), the cognitive accessibility of rape myths was manipulated in the present study through the completion of the RMA measure prior to that for rape proclivity.

The Bohner et al., ${ }^{28}$ study demonstrated that participants who endorsed elevated levels of RMA indicated higher proclivity to rape. However, a limitation was that developmental factors which contributed to the endorsement of RMA and higher levels of selfreported proclivity to rape were not assessed. In addition, the study also did not account for sexual aggression, through the likelihood to use force. Generally, early life experiences, acceptance of rape myths, hostility towards women and acceptance of interpersonal violence are themes which have been shown to be related to sexual aggression toward women. ${ }^{78,81}$ Interestingly, early life experiences related to an increased propensity towards sexual aggression are similar to those associated with adult psychopathology and dysfunctional cognitive processes. ${ }^{107}$ These dysfunctional cognitive processes reportedly are driven by early maladaptive schemas (EMSs) and make sexual offending more likely as they bias information processing through RMA. ${ }^{107}$ Developmental and psychological factors, in the form of EMSs, related to the likelihood to use force and rape (i.e., interchangeably referred to as self-reported proclivity to rape in the current paper) were explored in the current research project. In summary, the present study aimed at first, evaluating whether the relationship between RMA and self-reported proclivity to rape, as observed in Western societies, held true in Singapore. The current project also aimed at identifying whether there is an increased propensity towards acknowledging the likelihood to use force rather than rape among non-incarcerated men. Secondly, the study aimed at expanding on previous research that evaluated this increased proclivity to rape through evaluating the enduring developmental and biological factors (i.e., EMSs) that drive surface level cognitions, belief systems and behavioural inclinations. In view of this, the current study is divided into primary and secondary exploratory hypotheses.

\section{Primary hypotheses}

The primary hypotheses aimed at reviewing the relationship between RMA and the proclivity to rape women in a Singaporean college student sample. It was anticipated that participants who endorsed higher levels of RMA would report higher proclivity to rape. Subsequently, it was anticipated that the participants would report an increased tendency to use force rather than raping.

Relationship between RMA and the proclivity to rape: The current findings revealed that elevated levels of RMA predicted an increased self-reported proclivity to rape. As such, the hypothesis was supported and revealed that a positive correlational relationship between RMA and self-reported proclivity to rape held true in the Singaporean student sample. However, the assumption for normality was violated and subsequently converted with a log transformation to create a best fit model.The cause for non-normality does not appear to be due to data entry error, non-declared missing values or the presence of outliers. It is likely that normality was violated simply due to the exploratory nature of the research, coupled with the fact that the sample size is not extremely large. Non-normality appears to be a common dilemma in educational and psychological research. ${ }^{123}$ Osborne ${ }^{130}$ explained that a possible reason why the assumption normality of variables is not fulfilled is because the values do not spread over a large range with a large sample size. Moreover, given that the present study is exploring extreme behavioural patterns, the results are expected 
to skew to a certain side. This trend is of significant relevance due the employment of a non-clinical student sample. A majority of the current participants attained rather low scores, undeniably resulting in a skewed distribution.

Furthermore, in an effort to correct for the limitation of not controlling for social desirability in the first experiment of Bohner et al. ${ }^{28}$ study, the data was consecutively analysed while controlling for social desirability. However, the findings revealed that social desirability was not significantly related to predicting self-reported proclivity to rape. Henceforth, only findings that did not control for social desirability were included in the reported analyses. The absence of the relationship between social desirability and self-reported proclivity to rape may be suggestive of three possibilities. In the first place, the relationship between RMA and rape proclivity may have changed from significant to insignificant after the inclusion of the control variable (i.e., social desirability) due to the fact that both RMA and social desirability possibly accounted for similar variances. Secondly, it is important to consider that the only variable that appeared to be influenced by the propensity to respond in a socially desirable manner was RMA. The administration order of the measures may have also prompted these differential social desirability motives. Lastly, based on the poor internal consistency of the MCSDS when utilized with the current sample, it is likely that the measure did not sufficiently and accurately assess social desirability.

Moreover, although the predictive power of the analysis is rather small, it important to note that low R-squared values are seen in certain fields. Particularly, the predictive power in psychological research is often lower than 20 percent. ${ }^{131}$ Even despite the natural tendency for the predictive power to be small in psychological research, the existing variance of 4.3 percent is significantly smaller than that attained by Bohner et al., ${ }^{28}$ which was 23 percent. This proposes that the relationship between RMA and self-reported proclivity to rape in the current sample is not as strong as that in the previous research conducted in other countries. A smaller effect size in the relationship between RMA and proclivity to rape may be due to four possibilities. First and foremost, it is highly probable that the rape myths assessed in the present study may not be prominent in Singapore. RMA is likely to be among many other factors, which were not assessed in the present study, that predict self-reported rape proclivity in Singapore. These possibilities were corroborated by analysis of the general performance of the present sample on the AMMSA, whereby the mean score was revealed to be within the neutral range. Given that previous research on the relationship between RMA and proclivity to rape has primarily been done with college samples, the influence of education and age are unlikely to be confounding factors. Therefore, this suggests that the acceptance of rape myths is unlikely to be high in Singapore.

Secondly, the order of presentation of the measures may have influenced the accessibility and acknowledgement of rape myths and behavioural inclinations. Behavioural expressions of propensity towards sexual coercion and aggression have been forecasted to be differentially affected by strengthening the accessibility of preexisting attitudes. ${ }^{115}$ Thomas et al., ${ }^{115}$ further reiterated that cognitive factors such as RMA, which are often assessed on a trait level, have been shown to be significantly related to self-reported rape proclivity. However, this level of association is known to fluctuate on a state or situational basis. This viewpoint was first proposed by Schwarz et al., ${ }^{132}$ and validated by Bohner et al., ${ }^{28}$ As such, since it has been demonstrated through previous research that the activation of rape myths rendered higher endorsement of behavioural inclinations of rape, ${ }^{28}$ the measure of RMA was always presented before the measure of proclivity to rape in the current study. However, the first inventory completed was the YSQ-S3. It can be hypothesized that completing the YSQ-S3 may have manipulated the mood and thought processes of the participants, such that it influenced their ratings on the RMA measure and ASA-R. The completion of the YSQ-S3 may have influenced their performance on the RMA measure and ASA-R through heightening the participants' awareness of their present unmet needs and consequential emotional difficulties.

Thirdly, another possible explanation for the present outcomes may be that most of previous research on the subject matter of the association between rape myths, proclivity to rape and display of sexually aggressive behaviours are based on correlational studies. Some studies have attempted to take a more experimental approach, perhaps through the use of analogue studies by affording the opportunity for male participants who have elevated proclivity to rape and sexually coerce, to display some of their behavioural aggression within a laboratory setting. ${ }^{115}$ However, there appears to be a gap in research related to demonstrating the causal effect between cognitive sets (i.e., such as RMA and hostility towards women), proclivity to rape and sexually aggressive behaviours. The rationalization for the role of RMA in the development of sexually aggressive behaviours has been one that is contradictory. It is clear that individuals with higher acceptance of rape myths have an elevated proclivity to rape, and concomitantly display more sexually aggressive behaviours. Most studies demonstrated that these three concepts are correlated. ${ }^{115,133}$ This appreciation leads to the next question regarding the progression of RMA, which has been postulated to both follow ${ }^{134}$ and precede sexually aggressive behaviours. According to Chiroro et al., ${ }^{82}$ the anticipated level of enjoyment of sexual dominance appears to be modulated by pro-rape attitudes. Conversely, some researchers have suggested that rape myths may serve to rationalize and justify previous behaviour. ${ }^{28,135}$ As such, it is likely that the current findings are complicated due to the uncertainty in the development and concomitant role of the RMA.

Lastly, it is conceivable that rape myths in Singapore may vary from those in the West. Sexual aggression has not been avidly researched in Singapore or even within Asia. Much information related to this topic matter is derived from information pertained from explorations primarily in the West or countries which are culturally different from Singapore. While culture or ethnicity was not investigated as a variable in the present study, it is probable that the variability in the Western (i.e., the RMA measure used in the present study was developed in the West) and Asian culture may account for a portion of the divergence in the findings. Thomas and Gorzalka ${ }^{115}$ verified that the ways in which various variables interact to impact a particular behaviour, such as sexual coercion, is not expected to be culturally uniform. Due to limited research on this subject within Asia, particularly Singapore, information available from previous explorations in North America evaluating ethnicity may be utilized to understand the influence of culture on the present outcomes. While research on tolerance of sexual harassment in terms of coercive attitude among Asian and non-Asian individuals exists in North America, researchers have commented that there are still areas to be explored. ${ }^{136}$ Despite the existence of explorations of sexual aggression among men from Asian and Caucasian backgrounds in North America, ${ }^{115,136}$ this information ought to be evaluated with caution. Available knowledge may not necessarily generalize across diverse Asian cultures. Singapore, being composed of expatriates and citizens with roots from Indian, Malay, Chinese and European societies, is undoubtedly culturally rather unique and different from the societies where such cross cultural research has been conducted.

Investigations in North America revealed no significant differences 
in the incidence of sexual assault in terms of ethnicity ${ }^{137,138}$ However, the figure of reported rape cases are modulated by ethnicity. ${ }^{115}$ Asian women are less likely to report sexual assault, even disclosing the act to family and friends. ${ }^{139,140}$ The under-reporting sexual assault among Asian women may imply that beliefs supportive of sexual assault are likely to vary and fluctuate due to culture. ${ }^{115}$ Some theorists have further proposed that RMA is not ample to identify individuals at risk for sexual perpetration. Unique cultural factors appear to differentially influence the determinants of sexual aggression explored in the introduction section ${ }^{115}$. Disparities in consequences and motivations for aggressive acts among the collectivist and individualist societies have been long established. These acts are perceived to disintegrate social integrity, causing a loss of honour in the eyes of society and simply, deemed as crimes against society. ${ }^{141}$ More recently, Zane et al., ${ }^{142}$ substantiated that the concerns related to the loss of honour and integrity in the perceptiveness of society, are more salient in Asian men who associate with collectivism. Furthermore, in the event that there is a concern about losing face, sexually aggressive behaviours are likely to be inhibited. This understanding then brings us to a juncture, where the following questions arise: Do Singaporean males identify with collectivism or individualism? Does the consequence of "losing face" and perception of rape being a crime against society, in combination to traditional view that access to one's wife is absolute deter Singaporean men from stranger or acquaintance rape but unassumingly manifests in marital rape? While these questions have not been addressed in the present study, these factors may be contributing the present findings. Henceforth, information from existing research may aid in understanding the possible influence of these factors.

The definition of a core family unit in Singapore varies from that in the West. Here, the immediate family unit comprises of parents, children (adult and non-adult), mother's parents and siblings, and their children, and their children's children; and father's parents and siblings, and their children, and their children's children. ${ }^{143}$ Family is regarded as the core of society in Singapore. As traditional Asian values of familial ties continue to be prominent to the average Singaporean, the society is more collectivistic. ${ }^{144,145}$ Henceforth, it is probable that the consequences and motivations for acts of aggression among collectivistic societies may be related to the Singaporean context and explain the low RMA in the present study. Even if the latter of the above mentioned questions is true, a threat to society is still posed. This suggests that the same problems related to rape globally still do apply within the Singaporean context but, have been focused on the institution of marriage. To date, there is no publically available data for marital rape in Singapore, particularly as it is yet to be recognized as an act of rape. However, there is evidential support for a high incidence of marital rape in Singapore. A 2009 International Violence against Women survey conducted found that 71.7 percent of women abused by their partners were unwilling to lodge a formal police report. ${ }^{146}$ Rape myths and self-reported rape proclivity may exist within the context of marriage in Singapore. Henceforth, the lower RMA scores may be reflective of lesser acceptance for strange or acquaintance rape but, a greater acceptance for martial rape in Singapore. RMA was assessed more generally, in terms of violence against women and not related to marriage or intimate relationships with cohabiting partners, in the current study. Marital rape poses threats that are equivalent or greater to stranger or acquaintance rape. It raises issues related to public health and serious societal problems. ${ }^{147}$ Marital characteristics related to an augmented risk for marital rape include non-sexual marital aggression, low marital quality, status inequalities between spouses, and incessant disputes over finances, substance use, and sex. Victims of marital rape are often younger, tend experience rape outside of marriage, and are separated from their husbands. Meanwhile, risk factors for marital rape offenders include unemployment, hyper masculinity, and substance use, and sexual characteristics such as sexually coercive fantasies. ${ }^{147}$ Many of these qualities have previously been identified to be associated with rape perpetration. Marital rape impacts the family unit, the core of society, ${ }^{148}$ and concomitantly will have an influence both on an individual, in terms of psychological well-being, and societal level. Campbell et al., ${ }^{149}$ demonstrated that forced sex by an intimate partner is a crucial risk factor for homicide.

Propensity to report the use of force or rape: Furthermore, the current findings supported the second component of the primary hypotheses, which anticipated that men were more likely to report an increased tendency to use force rather than raping. This is evidenced by higher scores on items that assessed the likelihood to use force to get a woman to do something sexually she did not want to as compared to the likelihood to rape a woman. Malamuth et al., ${ }^{55}$ confirmed that while both items are highly correlated, they often render different responses. Akin to previous work, present findings corroborated that fewer men endorse the likelihood to engage in an act when the term "rape" is used.

This may reflect either the lack of acknowledgment regarding the similarities between using force or sexual coercion and rape, and the potential influence of rape myths. Rape myths appear to serve gender-specific functions. Denying engagement in behaviours defined as rape may allow men to distance themselves from the "bad" men who commit sexual assault, thereby serving as aprotective defensive mechanism. ${ }^{83}$ Moreover, the current rape proclivity measure did not distinguish between the use of physical force and sexual coercions. This permitted the participants to use their discretion to distinguish between the use of verbal pressure or physical force to coerce sexual interaction and rape, based on their personal interpretation of the constructs. Carr et al., ${ }^{150}$ demonstrated more college men conceded to sexual coercion as opposed to using physical force to obtain sex. Sexual coercion includes pressuring women and saying things they did not mean to obtain sex, using alcohol to obtain sex, and having sex with a woman even when she wanted to stop. Males are likely to associate rape with being related to use of physical force rather than sexual coercion. Individuals' definition of rape is influenced by rape myths ${ }^{78}$ Henceforth, since men are less prone towards viewing their efforts to coerce women into surrendering to their sexual advances as rape further supports the protective properties of rape myths.

\section{Secondary Hypotheses}

The secondary hypotheses were more exploratory in nature. Since childhood physical and emotional abuse, ${ }^{111,112}$ parental criminality ${ }^{44}$ and observing inter-parental aggression have been identified as risk factors for sexual offending, it was deduced that the disconnection and rejection schema domain may best describe the family of origin and developmental experiences of rape perpetrators and also individuals with elevated levels of proclivity to rape. Young ${ }^{62}$ highlighted that the focus ought to be on the five broad domains rather than individual EMSs. Since it has been acknowledged that men were more likely to indicate the likelihood to endorse items that use the term "force" rather than "rape", ${ }_{55,89,115}$ it was further hypothesized that the schemas endorsed by participants who report the likelihood to use force are not expected to differ from those who report the likelihood to rape, in terms of the types of schemas and domains endorsed.

Relationship between elevated proclivity to rape and schemas: The present findings revealed a final model containing three of the 18 schema predictors: Unrelenting Standards/Hyper-criticalness, 
Punitiveness and Abandonment/Instability. The model accounted for 12.3 percent of the variance of proclivity to rape. Elevated levels of proclivity to rape were primarily predicted by higher scores on the Unrelenting Standards/Hyper-criticalness schema, lower scores on the Punitiveness schema and to a lesser extent lower scores on the Abandonment/Instability schema. Unrelenting Standards/Hypercriticalness, Punitiveness and Abandonment/Instability schemas uniquely accounted for 24.8 percent, 26.7 percent and 18.1 percent of the variance of proclivity to rape respectively.

According to Onditi, ${ }^{131}$ low R-squared values are seen in fields such as psychology. Therefore, the present findings may be perceived as being prognostic and clinically relevant. The assumption that the disconnection and rejection domain may best describe the family of origin and developmental experiences of individuals with elevated self-reported proclivity to rape was only partially supported. Only the Abandonment/Instability schema is from the disconnection and rejection domain, however, this explains the least variance in an elevated self-reported proclivity to rape. The Unrelenting Standards/ Hyper-criticalness, which appears to have the most variance and Punitiveness schemas are from the Over-vigilance and Inhibition domain. Previous findings assessing EMSs among men attracted to sexual aggression is inconsistent. The current study contributes to this variability. Limited support for the secondary hypotheses and the evident variability in the present findings may be explained by two potential reasons. The first probable explanation is related to methodology. Such variability may reflect the difference between males attracted to sexual aggression and sex offenders. This is evidenced by the current findings being partially supportive of the conclusions drawn by Sigre et al., ${ }^{71}$ where males who reported sexual coercion of women endorsed higher levels of EMSs related to the disconnection and rejection, impaired autonomy/performance, and over-vigilance and inhibition domains. The focus is on the schema domains as it is anticipated that schemas within the same domains are correlated and serve similar purposes. Sigre et al. ${ }^{71}$ study is the only one that utilized a student sample. Henceforth, the present findings may possibly contribute preliminary support for the variability among sex offenders and men who have the proclivity to rape, in the terms of the family of origin and biological factors.

Another probable explanation might be the influence of culture. Although the role of culture was not explored as a variable in the current study, cultural difference is likely to be a factor contributing to the variable finding. While there may be some consistency when comparisons are made among studies conducted in similar cultures, the same conclusion cannot be drawn for studies conducted in varying societies. It has already been ascertained that EMSs are manipulated by culture. ${ }^{61}$ This view is further strengthened in the current study. Farr ${ }^{69}$ reported that rape perpetrators in North American endorsed higher levels of Instability/Abandonment and Defectiveness/Shame schemas, and only somewhat higher levels of the Emotional Deprivation schema (i.e., all three from Disconnection and Rejection domain) when contrasted with non-perpetrators. Varied EMSs were associated with predicting perpetration status. An increased propensity of being a rape perpetrator was associated with schemas from the Disconnection and Rejection domain (i.e., greater levels of emotional deprivation and defectiveness/shame schemas, and lowered levels of social isolation/alienation). Furthermore, higher levels of the insufficient self-control/self-discipline (i.e., impaired limits domain) and social isolation/alienation schemas were related to greater attraction towards sexual aggression. Conversely, a study by Manesh et al., ${ }^{70}$ in Iran revealed that apart from the disconnection/rejection domain, elevated performance on the impaired autonomy/performance domain was also observed among adult males with the propensity towards committing sexual offenses, some of whom were incarcerated sex offenders. Carvalho et al., ${ }^{65}$ added that rapists in Portugal presented with more schemas from the impaired autonomy/performance domains. All three studies were conducted with adult male sex offenders. Ethnicity was the main element that differed among the participants. In the present study, the recognition of the Abandonment/Instability schema, though to a smaller extent, among the current participants does suggest that the family of origin and developmental experiences of Singapore males with a higher self-reported proclivity to rape may be somewhat similar to those in the West. However, there appear to be other more significant factors which determine self-reported proclivity to rape in Singapore. This is evidenced by the over-vigilance and inhibition domain being seemingly more prominent.

Generally, the over-vigilance and inhibition domain reflects the expectancy of suppression of feelings and impulses, and the necessity to abide by rigid standards of performance and ethical behaviour. They appear to originate from grim and punitive families, where the emphasis is on performance, duty, following rules, hiding emotions, and avoiding mistakes. The unrelenting standards/ hyper-criticalness schema can be defined as the expectation to meet high internalized, inflexible standards of behaviour. Meanwhile, the punitiveness schema can be described by the expectation that mistakes ought to be harshly punished ${ }^{61}$ Given that individual schemas among the schema domains comprise of similar familial experiences and belief systems, it is believed that individual schemas amongst the domains are highly correlated. Schemas from the over-vigilance and inhibition domain may reflect the Asian society and parenting style and, also the unique motivators and factors associated with sexual aggression. Inhibition of anger and aggression is a prominent component of the emotional inhibition schema (i.e., from the over-vigilance and inhibition domain); ${ }^{44}$ This warrants the possibility that emotions associated with anger are not valued and perhaps ignored to meet the needs of others and/or rigid standards of behaviour determined by authority figures. Research has shown that there is variability in the common and valued emotions across cultures. Markus et al., ${ }^{151}$ conveyed that within individualist cultures, emotions such as anger and frustration are more common. Meanwhile, within collectivist societies, empathy and concern are largely valued. ${ }^{152}$

As previously explored, empathy deficits and a lack of concern for others is a core deficit among sex offenders through research in the West. ${ }^{42}$ Anger and frustration have been known to dis inhibit propensities towards sexual coercion and aggression, through supporting the perception that others are channel for the fulfilment of one's needs. ${ }^{115}$ Therefore, it may be established that anger plays a salient role in sexual aggression. However, the presentation of anger may vary across individualist and collectivist societies. It is probable that anger is more likely to be suppressed in collectivist societies. Moreover, insecure attachment, which is a developmental factor related to sexual aggression, is also associated with suppressed anger. ${ }^{153}$ Disorganized attachment has been shown to be correlated with sexual aggression and is said to stem from parenting styles which are frightening, resulting in a conflict for children as the usual source of security becomes one of fear. ${ }^{42}$ This appears to be reflective of the home environments that promote the development of schemas within the over-vigilance and inhibition domain. Although ethnicity and culture were not assessed as variables in the present study, based on previous research it can be assumed that the factors associated with sexual aggression are culturally specific. Asian parenting styles have been characterized by themes of family interdependence, concern over educational achievement, strictness and control. Parental authority 
is also perceptible among Asian families. ${ }^{154}$ Some researchers have argued that the notion of filial piety is associated with authoritarian moralism and parenting. ${ }^{155}$ Henceforth, offspring of Asian and authoritarian parents are encouraged to be submissive at the expense of their own desires. While parenting styles were not assessed in the present study, the prominence of authoritarian parenting within the Asian and even Singaporean context is clear. It is not uncommon for Singapore parents to use physical forms of punishment when disciplining their children. Moreover, it is also highly probable that the prominence of the over-vigilance and inhibition domain is reflective of the Singaporean society, where great emphasis is placed on following rigid rules determined by authority figures and harsh punishments for non-compliance. This may reinforce the previously discussed notion that rape is viewed as a crime against society in Asia. The harsh penalties for criminal offences in Singapore and also, the general need to comply with the rules set by authority figures may serve to deter males from perpetrating stranger and acquaintance rape, but not marital rape.

Variability in schema types among men with propensity to report the use of force or rape: Moreover, as predicted there was no difference among the men who endorsed the increased tendency to rape rather than use of force, for the Unrelenting Standards/Hypercriticalness, Punitiveness and Abandonment/Instability schemas. This supports the initial outcomes in the primary hypotheses and previous investigation that there is little difference among both groups of men. ${ }^{55,89,113}$

\section{Clinical implications}

The present study has demonstrated that apart from methodological issues, the possible influence of cultural factors and ethnicity is likely and imperative. It is clear that, while knowledge from existing research from the West is informative and can aid in the development of new avenues of research, it is important to review these findings within the context of one's own culture. Most of the research to date suggests that rape myths result in the distorted cognitions in support of sexual aggression and coercion. ${ }^{65}$ Apart from the influences of culture, the present findings continue to suggest that within the Singaporean context, rape myths determined from research in Western societies are likely to be only one small component among other unknown factors which increase the propensity towards selfreported proclivity to rape. The present outcomes may also largely reflect the impact of the Singaporean value system on the constructs assessed. Akin to other psychosocial problems, most of the available research is used not only to inform current mental health practitioners about the various processes at play but also to guide intervention. The same would most likely apply in the case of identifying individuals at risk for perpetration, intervention for sex offenders or even men who may have demonstrated sexual coercion. As such, it is crucial that continued efforts are made to identify these influential factors which increase the propensity towards self-reported proclivity to rape among Singaporean men, so that these can be addressed during treatment.

Moreover, Young's framework of EMSs has been recognized for affording a promising approach to better understanding, retrieving and modifying deep-seated maladaptive cognitive schemas of sexual offenders. ${ }^{71,156}$ Experts in the area of sexual aggression have acknowledged that understanding maladaptive beliefs and distorted cognitions, which are driven by EMSs, is a crucial constituent when exploring the causation of sexual aggression and offences. ${ }^{157}$ Beech et al., ${ }^{110}$ reinforced the need to address these factors, which underlie the surface level social skills deficits and negative emotions experienced by sexual offenders, during treatment. Attraction to sexual aggression is evidently related to negative early life events, which result in the development of EMSs. Presently, it has clearly been demonstrated that EMSs are related to proclivity to rape and also, are likely to vary on the basis of culture. Furthermore, while these outcomes continue to add the variability in the available knowledge, they may also highlight the difference among men who are solely attracted to sexual aggression in comparison to those who are prone to offending. The identification of the EMSs related to attraction to sexual aggression does not only clarify the environmental factors related to the development of such schemas, however also is relevant to better understanding recidivism. Carvalho et al. ${ }^{65}$ reinforced that EMSs offer insight into the psychological and social adjustment of offenders. This information is not biased by the post hoc justifications of the crime. The Young's framework of EMSs is also reflective of how the individual will cope with life's challenges. These coping mechanisms are known to preserve the EMSs through enabling the avoidance of experiencing distressing emotions accompanying the activation of the schemas. ${ }^{61}$ As such, this is an issue which is pertinent to risk management and preventing relapse of the negative emotions experienced by sexual offenders. ${ }^{66,67}$ In summary, the current findings provide plausible support for further research on the use of Schema Therapy ${ }^{61}$ in rehabilitating sexually aggressive behaviours.

Of paramount importance is the need to recognize that these early life events are not consistent, as evidenced by them varying across cultures. This knowledge is relevant to fields of clinical and forensic psychology. From a clinical perspective, understanding the above mentioned unique factors are crucial during therapeutic intervention, as they would unexpected feed the clinical formulation and impact the effectiveness of therapeutic programs. From a forensic perspective, targeting these crucial factors during mandatory treatment is likely to influence rates of recidivism among sex offenders. Moreover, the present findings revealed the possibility that varied EMSs are endorsed by men with an attraction towards sexual aggression and sex offenders. As aforementioned, this information may be imperative during forensic risk assessments. Finally, the present findings are also relevant to academia as they emphasize the need for continued investigations. Future research on this subject matter in Singapore ought to build on this study and consider the role cultural differences and methodical errors highlighted in this section. More thorough research on the use of Schema Therapy in the rehabilitation of male with the propensity towards sexual aggression or history of sexual offending, is also recommended.

\section{Limitations of current research and future directions}

Firstly, an obvious constrain of the present study is the nonfulfilment of the assumptions for the data analyses. While some corrective measures in the form of data transformations were taken, the current data was still largely skewed. The assumptions for the secondary hypotheses were also not evaluated. This was primarily due to the fact that these analyses were exploratory in nature. Furthermore, the data is anticipated to be skewed when extreme behaviour is examined. Micceri ${ }^{123}$ found that only a minority of the data collected in educational and psychological research follows univariate normal distributions. As such, it is advisable to interpret the current findings with caution and perhaps, to correct for the statistical errors of the primary hypothesis through the use of a larger sample size in future investigations. Secondly, another limitation of the current study was the use of a convenient student sample. While the benefits of using a sample of non-incarcerated men when attempting to investigate self-reported rape proclivity have been acknowledged by experts in 
this area and also previously discussed in the former sections, the current findings are limited to the males at an international university in Singapore. It is difficult to conclude if these outcomes can be generalized to the Singaporean male population as a whole. Moreover, the order in which the questionnaires were completed may raise the previously explored issue of trait versus state impact of attitudes and behavioural inclinations measured. As such, it is recommended that future research is expanded to include males from the general society. It may be worthwhile to replicate the present study with men from the general population and incarcerated sex offenders. Researchers keen to further explore this subject matter may also wish to consider experimentally manipulating the order in which the YSQ-S3 is administered to determine if it has an impact on the RMA measure and ASA-R.

Thirdly, the current findings have demonstrated that while there is a similar trend in the relationship between RMA and selfreported proclivity to rape, the effect size of this relationship was smaller than that attained by Bohner et al. ${ }^{28}$. Thereby suggesting that rape myths may either be less prominent in Singapore in terms of determining self-reported rape proclivity or different from those identified through research in the West. This highlights the existence of cultural differences among Asian and Western societies. Such dissimilarities may be even more apparent in the Singaporean society, due to Singapore's vast cultural make-up and divergence from other Asian societies in the region. Henceforth, it may be worthwhile to consider other assumptions made by Western researchers regarding studying sexual aggression and rape. One of such assumptions is that incarcerated sex offenders may not adequately represent men within the generally society who are sexually aggressive. As such, it would be recommended that researchers eager to expand the current study consider including a comparison between men in the general population who are attracted to sexual aggression and incarcerated sex offenders.

Fourthly, it is established that rape myths can be conceived as cognitive schemas, ${ }^{158}$ and acquaintance rape is more common than stranger rape (FBI, 2000). The reframing of the view that it is acceptable for a man to force his wife to have sex with him, to that being an act of rape is also a fairly new development in Western society. The right to access one's wife has often been accepted as being absolute. ${ }^{159,160}$ The first martial rape case was trialled in the American court system in 1978. Only in 1993, was marital rape recognized as a crime in all the 50 states in America. However, in 30 of these states, exemptions may be given to husbands from a rape prosecution. ${ }^{159-161}$ Given that marital rape is not viewed or culturally accepted as rape in Singapore, the current findings may be further implicated by the possibility that the men in the current sample who endorsed higher levels of rape proclivity and RMA may be those who are vulnerable or accepting of stranger rape. This is primarily because the AMMSA did not distinguish the relationship between the perpetrator and victim. The current findings also did not account for the men who are willing to inflict sexual aggression on their wives or intimate partners. It would be interesting to see if the acceptance of marital or intimate relationship rape myths are related to a higher selfreported proclivity to rape, and if the effect size of this relationship is larger when the relationship between the perpetrator and victim is not left ambiguous as in the current measure of RMA used (i.e., AMMSA). Assuming that a difference exists when the relationship between the perpetrator and victim is made known to be one that is intimate, further investigation into identifying the EMSs of this group of individuals would be recommended. Lastly, methodological and procedural errors may have influenced the present findings. These errors are related to the length of the questionnaire battery and data collection sessions. Data was collected through the forum of group sessions. It may be likely that this setting may have influenced the participants' sense of confidentiality and therefore, biased their responses. The plausibility of using online survey sessions may be worthwhile to consider in future research. Additionally, the ASA-R may not have adequately assessed self-reported proclivity to rape. It is likely that due to the length of the current questionnaire battery (i.e., which took 45 minutes to an hour to complete) provided to the participants in the current study, a majority of them may have become fatigued with large number of questions and eventually, unmotivated to provide accurate responses. Some alternatives for other studies attempting to replicate the current research in Singapore may include the employment of other material that has been indicated be less wellstructured but of similarly high face validity, with regard to assessing the social-cognitive functions of RMA. These may include the use of true-to-life and convincing photographs or video vignettes in various rape scenarios. The application of visual stimuli also seems to correct for some of the limitations of rape scenarios, which were previous discussed. ${ }^{158}$ Meta-analyses on this subject matter have revealed sexual arousal to scenes of sexual coercion involving force appears to be varied between convicted rapists and non-rapists, with the former demonstrating higher levels of arousal. ${ }^{162-164}$ However, while the application of the visual stimuli may appear corrective, one ought to recognize that this mode of evaluation also has its own confines. For instance, rapists and child molesters seem to be able to disinhibit their sexual arousal to their preferred stimuli but also, generate arousal to non-preferred and socially acceptable stimuli. ${ }^{165-170}$

\section{Conclusions}

The current study is among the first to explore RMA and EMSs within the Singaporean context and has provided a platform for future investigations into this matter. It clearly emphasizes the possibility that factors other than RMA are relevant within the Singaporean context when determining self-reported proclivity to rape. These factors seem to be associated with the Asian culture, and appear to even influence the EMSs related to an elevated proclivity to rape. Furthermore, it appears that the rape myths prevalent in Singapore may not be akin to those in Western societies. Identifying the stereotypes surrounding rape within the current cultural context is important, especially when attempts are made to challenge and educate society about these stereotypes. Understanding the above mentioned unique factors is crucial during therapeutic intervention. This would feed the clinical formulation, impact the effectiveness of therapeutic programs and influence rates of recidivism among sex offenders. Future research on this subject matter in Singapore ought to build on this study and consider the role cultural differences and methodical errors highlighted in this section. Lastly, the present finding also corroborates preliminary support for more thorough research on the use of Schema Therapy in the rehabilitation of males with the propensity towards sexual aggression or history of sexual offending. As discussed prior, although EMSs are not specific to offenders' impressions about their victims or perception of their crimes, they offer a wealth of information related to both the self-perception and world view of sex offenders, and consequentially reflecting their level of psychological and social adjustment. ${ }^{65}$ Schema Therapy offers insight into the offender's world view and how he copes with life's challenges. It is expected that there will be an influence of recidivism through the exploration of these topics in treatment and utilization of this available information to discuss relapse prevention.

\section{Acknowledgements}

None. 


\section{Conflicts of interest}

Author declares there are no conflicts of interest.

\section{Funding}

None.

\section{References}

1. Norfolk GA. Leda and the swan-and other myths about rape. J Forensic Leg Med. 2011;18(5):225-232.

2. Gerger H, Kley H, Bohner G, et al. The acceptance of modern myth about sexual aggression scale: Development and validation in German and English. Aggressive Behaviour. 2007;33(5):422-440.

3. Kulkarni J. Women and mental health. Australian Women's Health Network. 2012

4. Ellsberg M, Heise L, World Health Organization, Program for Appropriate Technology in Health. Researching violence against women: A practical guide for researchers and activists. Geneva: WHO; Washington DC: PATH. 2005

5. United Nations .Declaration on the Elimination of Violence against Women. United Nations General Assembly, New York, USA. 1993.

6. Basile K, Saltzman L. Sexual violence surveillance uniform definitions andrecommended data elements version 1.0. Atlanta: Centers for Disease Control and Prevention, National Center for Injury Prevention and Control. 2002.

7. Hardit S. Predicting sexual aggression among college men: The role of male peer groups and sexualized media (unpublished doctoralispepdissertation). University of Illinois, IL, United States. 2002.

8. Faúndes A, Andalft J. Sexual violence against women. the role of gynecology and obstetrics societies in Brazil. International Journal of Gynecology and Obstetrics. 2002;78(1):S67-S73.

9. Baumer EP, Felson RB, Messner SF. Changes in police notification for rape, 1973-2000. Criminology. 2003;41(3):841-872.

10. Bouhours $\mathrm{B}$, Cheong $\mathrm{CW}$, Bong $\mathrm{B}$, et al. International violence against women survey: Final report on Singapore. 2013.

11. National Intimate Partner and Sexual Violence Survey. Summary Report. 2010

12. United Nations. Fact Sheet on Violence Against Women. 2008.

13. Zawacki T, Abbey A, Buck PO, et al. Perpetrators of alcohol-involved sexual assaults: How do they differ from other sexual assault perpetrators and non-perpetrators. Aggressive Behaviour. 2003;29(4):366-380.

14. Garcia Moreno C. Violence against women: International perspectives. American Journal of Preventive Medicine. 2000;19(4):330-333.

15. Wolitzky-Taylor KB, Resnick HS, McCauley JL, et al. Is reporting of rape on the rise? A comparison of women with reported versus unreported rape experiences in the national women's study-replication. J Interpers Violence. 2011;26(4):807-832.

16. Marshall WL, Barbaree HE. A behavioural view on rape. Int $J$ Law Psychiatry. 1984;7(1):51-77.

17. Association of Women for Action and Research (AWARE). Convention on the Elimination of All Forms of Discrimination against Women (CEDAW) shadow report. 2011

18. Patterson D, Campbell $\mathrm{R}$. Why rape survivors participate in the criminal justice system. Journal of Community Psychology. 2010;38(2):191-205.

19. Edwards KM, Turchik JA, Dardis CM, et al. Rape myths: History, individual and institutional-level presence, and implications for change. Sex Roles. 2011;65(11):761-773.
20. Stubbs J. Sexual assault, criminal justice and law and order. Women Against Violence: An Australian Feminist Journal. 2003;(14):14-26.

21. Whatley MA. Victim characteristics influencing attributions of responsibility to rape victims: A meta-analysis. Aggression and Violent Behaviour. 1996;1(2):81-95.

22. Clark H. Judging rape. ACSSA Newsletter. 2007;14:22-23.

23. Easteal P, Bartels L, Bradford S. Language, gender and 'reality': Violence against women. International Journal of Law, Crime and Justice. 2012;40(4):324-327.

24. Jones JS, Alexander C, Wynn BN, et al. Why women don't report sexual assault to the police: The influence of psychosocial variables and traumatic injury. J Emerg Med. 2009;36(4):417-424.

25. Abel GG, Becker JV, Blanchard EB, et al. The behaviour assessment of rapist. In J. Hays, T. Roberts \& K. Solway (Eds.), Violence and the violent individual. Holliswood, Spectrum Publications, New York, USA. 1981 .

26. Barbaree HE, Marshall WL. The Role of male sexual arousal in rape: Six models. Journal of Consulting and Clinical Psychology. 1991;59(5):621-630.

27. Lussier P, Cale J. Beyond sexual recidivism: A review of the sexual criminal career parameters of adult sex offenders. Aggression and Violent Behaviour. 2013;18(5):445-457.

28. Bohner G, Reinhard M, Rutz S, et al. Rape myths as neutralizing cognitions: Evidence for a causal impact of anti-victim attitudes on men's self-reported likelihood of raping. European Journal of Social Psychology. 1998;28(2):257-268.

29. Ward T, Beech AR. An integrated theory of sexual offending. Aggression and Violent Behaviour. 2006;11(1):44-63.

30. New A, Goodman M, Mitropoulou V, et al. Genetic polymorphisms and aggression. In J Benjamin \& Ebstein RP (Eds.), Molecular genetics and the human personality. American Psychiatric Publishing, Washington, DC, USA. 2012;231-244.

31. Baumeister RF, Catanese KR, Vohs KD. Is there a gender difference in strength of sex drive? Theoretical views, conceptual distinctions, and a review of relevant evidence. Personality and Social Psychology Review. 2001;5(3):242-273.

32. Coccaro EF, Kavoussi RJ, McNamee B. Central neurotransmitter function in criminal aggression. In: Fishbein (Ed.), The science, treatment, and prevention of antisocial behaviours: Application to thecriminal justice system (pp. 1-16). Kingston, NJ: Civic Research Institute. 2000.

33. Abbey A, Zawacki T, Buck PO, et al. Alcohol and sexual assault. Alcohol Research \& Health: The Journal of the National Institute on Alcohol Abuse and Alcoholism. 2001;25(1):43-51.

34. Brecklin LT, Ullman SE. The roles of victim and offender alcohol use in sexual assaults: results from the national violence against women survey. J Stud Alcohol. 2002; 63(1):57-63.

35. Baron L, Straus MA. Four theories of rape: A macrosociological analysis. Social Problems. 1987;34(5):467-489.

36. Linz D. Exposure to sexually explicit materials and attitudes toward rape: A comparison of study results. The Journal of Sex Research. 1989;26(1):50-84.

37. Malamuth N. Aggression against women: cultural and individual causes. In Donnerstein E (Eds.), Pornography and sexual aggression. Academic Press, Orlando Florida, USA. 1984.

38. Malamuth N. Predictors of naturalistic sexual aggression. J Pers Soc Psychol. 1986;50(5):953-962.

39. Sanders T, Campbell R. Designing out vulnerability, building in respect: Violence, safety and sex work policy. Br J Sociol . 2007;58(1):1-19. 
40. Nixon K, Tutty L, Downe P, et al. The everyday occurrence: Violence in the lives of girls exploited through prostitution. Violence Against Women. 2002;8(9):1016-1043.

41. Dwyer S. Pornography. In: Livingstone, Plantinga (Eds.), The routledgecompanion to philosophy and film. Routledge, New York, USA. 2008.

42. Burk LR, Burkhart BR. Disorganized attachment as a diathesis for sexual deviance: Developmental experience and the motivation for sexual offending. Aggression and Violent Behaviour: A Review Journal. 2003;4:487-511.

43. Marshall WL, Marshall, LE. The origins of sexual offending. Trauma, Violence and Abuse. 2000;1(3):250- 263.

44. Richardson G. Early maladaptive schemas in a sample of british adolescent sexual abusers: Implications for therapy. Journal of Sexual Aggression. 2005;11(3):259-276.

45. Straus MA, Gelles RJ, Steinmetz SK. Behind closed doors: Violence in the American family. Sage Publications, Newbury Park, California, USA. 1988.

46. Cale J, Lussier P. Merging developmental and criminal career perspectives: Implications for risk assessment and risk prediction of violent/sexual recidivism in adult sexual aggressors of women. Sexual Abuse: A Journal of Research and Treatment. 2012;24(2):107-132.

47. Cortoni F, Marshall WL. Sex as a coping strategy and its relationship to juvenile sexual history and intimacy in sexual offenders. Sexual Abuse: A Journal of Research and Treatment. 2001;13(1):27-43.

48. Marshall WL, Barbaree HE. An integrated theory of sexual offending. In DR Laws (Eds.), Handbook of sexual assault: Issues, theories, and treatment of the offender. Plenum press, New York, USA. 1990;257-275.

49. Seidman BT, Marshall WL, Hudson SM, et al. An examination of intimacy and loneliness in sex offenders. Journal of Interpersonal Violence. 1994;9(4):518-534.

50. Malamuth NM. Rape proclivity among males. Journal of Social Issues. 1981;37(4):138-157.

51. Ward T, Keenan T, Hudson SM. Understanding cognitive, affective and intimacy deficits in sexual offenders: A developmental perspective. Aggression and Violent Behaviour. 2000;5(1):41-62.

52. Wood RM, Grossman LS, Fichtner CG. Psychological assessment, treatment, and outcome with sex offenders. Behav Sci Law. 2000;18(1):23-41.

53. Kirsch LG, Becker JV. Sexual offending: Theory of problem, theory ofchange, and implications for treatment effectiveness. Aggression and Violent Behaviour. 2006;11(3): 208-224.

54. Polaschek DL, Ward T, Hudson SM. Rape and rapists: Theory and treatment. Clinical Clin Psychol Rev. 1997;17(2):117-144.

55. Malamuth NM, Dean KE. Attraction to sexual aggression. In Parrot A \& Bechofer L (Eds.), Acquaintance rape: The hidden crime. New York, Wiley, USA. 1991;229-247.

56. Lim S, Howard R. Antecedents of sexual and non-sexual aggression in young Singaporean men. Personality and Individual Differences. 1998;25(6):1163-1182.

57. Malamuth N. Criminal and noncriminal sexual aggressors: Integrating psychopathology in a hierarchical-mediational confluence model. Ann N Y Acad Sci. 2003;989:33-58.

58. Voller EK, Long PJ, Aosved AC. Attraction to sexual violence towards women, sexual abuse of children, and non-sexual criminal behaviour: Testing the specialist vs. generalist models in male college students. Arch Sex Behav. 2009;38(2):235-243

59. Lalumiere ML, Harris GT, Quinsey VL, et al. The causes of rape. Washington, DC:AmericanPsychological Association. 2005.
60. Welburn KR, Dagg P, Coristine M, et al. Schematic change as a result of an intensive group-therapy day-treatment program. Psychotherapy: Theory, research, practice, training. 2000;37(2):189-195

61. Young JE, Klosko JS, Weishaar ME. Schema therapy: A practitioner's guide. The Guilford Press, New York, USA. 2003.

62. Young JE. Cognitive Therapy for Personality Disorders: A Schemafocused Approach. Professional Resource Exchange, Sarasota, FL, USA. 1990

63. Young JE. Cognitive therapy for personality disorders: A schema focused approach (Rev. ed.). Professional Resources Press, Sarasota, Florida, USA. 1999.

64. Riso LP, McBride C. Introduction: A return to focus on cognitive schemas. In DJ Stein and JE Young (Eds.), Cognitive schemas and core beliefs in psychological problems: A scientist-practioner guide. American Psychological Association, Washington, DC, USA. 2007

65. Carvalho J, Nobre PJ. Early maladaptive schemas in convicted sexual offenders: Preliminary findings. Int $J$ Law Psychiatry. $2014 ; 37(2): 210-216$

66. Gannon TA, Wright DB, Beech AR., et al. Do child molesters hold distorted beliefs? What does their memory recall tell us? Journal of Sexual Aggression. 2006;12(1):5-18.

67. Maruna S, Mann, RE. A fundamental attribution error? Rethinking cognitive distortions. Legal and Criminological Psychology. 2006;11(2):155-177.

68. Thornton D. Constructing and testing a framework for dynamic risk assessment. Sexual Abuse: A Journal of Research and Treatment. 2002;14(2):139-153.

69. Farr RA. The relationship between attachment, early maladaptive schemas, and rape and sexual assault perpetration (unpublished doctoralisepidissertation). Universityof La Verne, CA, USA. 2010. p.220.

70. Manesh EH, Baf HRAMS, Abadi BAGHA, et al. Early maladaptive schemas and schema domains in rapists. Iranian Journal of Psychiatry and Clinical Psychology. 2010; 16(2):145-153.

71. Sigre-Leirós VL, Carvalho J, Nobre P (2013) Early maladaptive schemas and aggressive sexual behaviour: A preliminary study with male college students. J Sex Med 10(7): 1764-1772.

72. Hanson RK, Bussière MT. Predicting relapse: A meta-analysis of sexual offender recidivism studies. J Consult Clin Psychol. 1998;66(2):348-362.

73. Frances A, Wollert R. Sexual sadism: Avoiding its misuse in sexually violentpredator evaluations. Journal of the American Academy of Psychiatry and the Law. 2012;40(3):409-416.

74. Miller L. Rape: Sex crime, act of violence, or naturalistic adaptation? Aggression and Violent Behaviour. 2014;19(1):67-81.

75. Hanson RK, Morton Bourgon K .Predictors of sexual recidivism: An updated meta-analysis. Ottawa, Public Safety and Emergency Preparedness Canada. 2004.

76. Gilbert F, Daffern M, Talevski D, et al. The role of aggressionrelated cognition in the aggressive behaviour of offenders: A genera aggression model perspective. Criminal Justice and Behaviour. 2013;40(2):119-138.

77. Brownmiller S. Against our will: Men, women and rape. New York: Simon \& Schuster. 1975

78. Burt MR. Cultural myths and supports for rape. Journal of Personality and Social Psychology. 1980;38(2):217-230.

79. EysselF, Bohner G. Schema effects of rape myth acceptance on judgments of guilt and blame in rape cases: The role of perceived entitlement to judge. Journal of Interpersonal Violence. 2011;26(8):1579-1605.

80. Suarez E, Gadalla TM. Stop blaming the victim: A meta-analysis on rape myths. Journal of Interpersonal Violence. 2010;25(11):2010-2035. 
81. Ryan KM. Further evidence for a cognitive component of rape. Aggression and Violent Behaviour: A Review Journal. 2004;9(6):579-604.

82. Chiroro P, Bohner G, Viki GT, et al. Rape myth acceptance and rape proclivity: Expected dominance versus expected arousal as mediator in acquaintance-rape situations. Journal of Interpersonal Violence. 2004;19(4): 427-442.

83. Lonsway KA, Fitzgerald LF. Rape myths: In review. Psychology of Women Quarterly. 1994;18(2):133-164.

84. Kalichman SC, Simbayi LC, Kaufman M, et al. Gender attitudes, sexual violence, and HIV/AIDS risks among men and women in Cape Town, South Africa. J Sex Res. 2005; 42(4):299-305.

85. Lee J, Kim J, Lim H. Rape myth acceptance among Korean college students: The roles of gender,attitudes toward women, and sexual double standard. Journal of Interpersonal Violence. 2010;25(7):1200-1223.

86. Payne DL, Lonsway KA, Fitzgerald LF. Rape myth acceptance: Exploration of its structure and its measurement using the Illinois rape myth acceptance scale. Journal of Research in Personality. 1999;33(1):27-68.

87. Chapleau KM, Oswald DL. Power, sex, and rape myth acceptance: Testing two models of rape proclivity. J Sex Res. 2010;47(1):66-78.

88. Blumenthal S, Gudjonsson G, Burns J. Cognitive distortions and blame attribution in sex offenders against adults and children. Child Abuse \& Neglect. 1999;23(2):129-143.

89. Malamuth NM. The attraction to sexual aggression scale: Part one. The Journal of Sex Research. 1989;26(1):26-49.

90. Dean KE , Malamuth NM. Characteristics of men who aggress sexually and of men who imagine aggressing: Risk and moderating variables. Journal of Personality and Social Psychology. 1977;72(2):449-455.

91. Schewe PA, O’Donohue, W. Sexual abuse prevention with high-risk males: The roles of victim empathy and rape myths. Violence Vict. 1993;8(4):339-351.

92. Abrams D, Viki GT, Masser B, et al. Perceptions of stranger and acquaintance rape: The role of benevolent and hostile sexism in victim blame and rape proclivity. J Pers Social Psychol. 2003;84(1):111-125.

93. Bohner G, Jarvis CI, Eyssel F, et al. The causal impact of rape myth acceptance on men's rape proclivity: Comparing sexually coercive and noncoercive men. European Journal of Social Psychology. 2005;35(6):819-828.

94. Malamuth NM, Haber S, Feshabach S. Testing hypotheses regarding rape: Exposure to sexual violence, sex differences, and the "normality" of rape. Journal of Research in Personality. 1980;14(1):121-137.

95. Bohner G, Siebler F, Schmelcher J. Social normal and the likelihood of raping: Perceived rape myth acceptance of others affects men's rape proclivity. Personality and Social Psychology Bulletin. 2006;32(3):286-297.

96. Crowne DP, Marlowe D. A new scale of social desirability independent of psychopathology. Journal of Consulting Psychology. 1960;24(4):349-354.

97. Tang CSK, Critelli JW, Porter JF. Sexual aggression and victimization in dating relationships among Chinese college students. Arch Sex Behav. 1995;24(1):47-53.

98. Ward C. Attitudes toward male-female relationships and rape justification beliefs in Singapore. In Lagunes IR \& Poortinga YH (Eds.) From a different perspective: Studies of behaviour across culture. Swets and Zeitlinger NV, Netherlands. 1985.

99. Tang CSK, Critelli JW, Porter JF. Motives in sexual aggression. Journal of Interpersonal Violence. 1993;8(4):435-445.

100. Elliott JM, Tong CK, Tan PM. Attitudes of the Singapore public to actions suggesting child abuse. Child Abuse \& Neglect. 1997;21(5):445-464.
101. Meston CM, Heiman, JR, Trapnell, et al. Ethnicity, desirable responding and self-reports of abuse: A comparison of European- and Asianancestry undergraduates. J Consult Clin Psychol. 1999;67(1):139-144.

102. Back SE, Jackson JL, Fitzgerald M, et al. Child sexual and physical abuse among college students in Singapore and the United States. Child Abuse \& Neglect. 2003;27(11):1259-1275.

103. Daro D, Gelles RJ. Public attitudes and behaviours with respect to child abuse prevention. Journal of Interpersonal Violence. 1992;7(4):517-531.

104. Straus MA, Gelles RJ. Physical violence in American families: Risk factors and adaptations to violence in 8,145 families. Transaction Publisher, New Brunswick, New Jersey, USA. 1992.

105. Milner RJ, Webster SD. Identifying schemas in child molesters, rapists, and violent offenders. Sex Abuse. 2005;17(4):425-439.

106. Murphy WD. Assessment and modification of cognitive distortions in child molestors. In Marshall WL \& Barbaree HE (Eds.), Handbook of sexual assaults: Issues, theories and treatment of the offender. Plenum Press, New York, USA. 1991;331-342.

107. Mann RE, Beech AR. Cognitive distortions, schemas, and implicit theories. In Laws DR \& Hudson SM (Eds.), Sexual deviance: Issues and controversies. London Sage,UK. 2003;135-153.

108. Thimm JC. Personality and early maladaptive schemas: A five-factor model perspective. J Behav Ther Exp Psychiatry. 2010;41(4):373-380.

109. Shapour S, Ma R, Akbari B, et al. Comparison between early maladaptive schema with psychological resiliency of the criminals murder, drug trafficking, rape. Procedia - Social and Behavioural Sciences. 2013;84(9):1105-1109.

110. Beech AR, Bartels RM, Dixon L. Assessment and treatment of distorted schemas in sexual offenders. Trauma, Violence, \& Abuse. 2013;14(1):54-66.

111. McGee RA, Wolfe DA, Wilson, SK. Multiple maltreatment experiences and adolescent behaviour problems: Adolescents' perspectives. Dev Psychopathol. 1997;9(1):131-149.

112. Straus MA. Measuring intra family conflict and violence: The conflict tactics (CT) scales. Journal of Marriage and the Family. 1979;41(1):75-88.

113. Malamuth NM. The attraction to sexual aggression scale: Part two. The Journal of Sex Research. 1989;26(3):324-354.

114. Hall GC, Hirschman R. Toward a theory of sexual aggression: A quadripartite model. J Consult Clin Psychol . 1991;59(5):662-669.

115. Thomas LA, Gorzalka BB. Effect of sexual coercion proclivity and cognitive priming on sexual aggression in the laboratory. J Sex Res. 2013;50(2):190-203.

116. Prentky RA, KnightRA. Identifying critical dimensions for discriminating among rapists. J Consult Clin Psychol. 1991;59(5):643-661.

117. Young JE. Young Schema Questionnaire- Short Form 3 (YSQS3) Schema Therapy Institute, New York, USA. 2005.

118. Jovev M, Jackson HJ. Early maladaptive schemas in personality disorderedindividuals. J Pers Disord. 2004;18(5):467-478.

119. Reeves M, Taylor J. Specific relationships between core beliefs and personality disorder symptoms in a non-clinical sample. Clinical Psychology and Psychotherapy. 2007; 14(2):96-104.

120. Hawke LD, Provencher, Martin D. The Canadian French young schemaquestionnaire: Confirmatory factor analysis and validation in clinical and nonclinical samples. Canadian Journal of Behavioural Science/Revue Canadienne Des Sciences Du Comportement. 2012;44(1):40-49.

121. Saritas D, Gençöz T. Psychometric properties of "Young Schema Questionnaire-Short Form 3" in a Turkish adolescent sample. Journal of Cognitive and Behavioural Psychotherapies. 2011;11(1):83-96. 
122. Simona T. The Roman version of the Young Schema Questionnaire Short Form 3 (YSQ- S3). Journal of Evidence-Based Psychotherapies. 2006;6(2):173-181.

123. Micceri T. The unicorn, the normal curve, and other improbable creatures. Psychological Bulletin. 1989;105(1):156-166.

124. Tabachnick BG, Fidell, LS. Multiple regression. In Using multivariate statistics, (5th edn.), Pearson/A\&B, Boston, Mass, Sydney. 2007.

125. McKibbin WF, Shackelford TK, Goetz AT, et al. Why do men rape? An evolutionary perspective. Review of General Psychology. 2008;12(1):86-97.

126. Gannon TA., Collie RM, Ward T, et al. Rape: Psychopathology, theoryand treatment. Clin Psychol Rev. 2008;28(6):982-1008.

127. Wolf N. The Beauty Myth. William Morrow, New York, USA. 1991.

128. Lisak D, Miller PM. Repeat rape and multiple offending among undetected rapists. Violence Vict. 2002;17(1):73-84.

129. Eyssel F, Bohner G, Siebler F. Perceived rape myth acceptance of others predicts rape proclivity: Social norm or judgmental anchoring? Swiss Journal of Psychology. 2006;65(2):93-99.

130. Osborne J. Notes on the use of data transformations. Practical Assessment, Research \& Evaluation. 2002; 8(6):1-8.

131. Onditi AA. Relationship between customer personality, service features and customer loyalty in the banking sector: A survey of banks in homabay county, Kenya. International Journal of Business and Social Science . 2013;4(15):132-150.

132. Schwarz N, Strack F. Manipulating salience: Causal assessment in natural settings. Personality and Social Psychology Bulletin. 1981;7(4):554-558

133. Malamuth NM, Sockloskie RJ, Koss MP, et al. Characteristics of aggression against women: testing a model using a national sample of college students. J Consult Clin Psychol. 1991;59(5):670-681.

134. Drieschner K, Lange A. A review of cognitive factors in the etiology of rape: Theories, empirical studies and implications. Clinical Psychology Review. 1999;19(1):57-77.

135. Prah M, Ayerakwa MM. How rape offenders view their crimes: A study of offenders in selected police cells and the central prison in Kumasi, Ghana. IFE-Psychologia: An International Journal. 2001;9:193-205.

136. Kennedy MA, Gorzalka BB. Asian and non-Asian attitudes toward rape, sexual harassment, and sexuality. Sex Roles. 2002;46(7):227-238.

137. Hall GCN, Sue S, Narang DS, et al. Culture-specific models of men's sexual aggression: Intra- and interpersonal determinants. Cultural Diversity and Ethnic Minority Psychology. 2000;6(3):252-267.

138. Kalof L. Ethnic differences in female sexual victimization. Sexuality and Culture. 2000;4(4):75-98.

139. Koss MP, Gidycz CA, Wisniewski N.The scope of rape: Incidence and prevalence of sexual aggression and victimization in a national sample of using social norms to reduce men's rape proclivity highereducation students. Journal of Consulting and Clinical Psychology. 1987;55(2):162-170.

140. Tjaden P, Thoennes N. Extent, Nature, and Consequences of Rape Victimization: Findings. The National Violence against Women Survey. National Institute of Justice, Washington, DC, USA. 2006.

141. Hall G, Barongan C. Prevention of sexual aggression: Sociocultural risk and protective factors. American Psychologist. 1997;52:5-14

142. Zane N, Mak W. Major approaches to the measurement of acculturation among ethnic minority populations: A content analysis and an alternative empirical strategy. In Acculturation: Advances in theory, measurement, and applied research. American Psychological Association, Washington, DC, USA. 2003;39-60.
143. Craig, Jo Ann. Culture shock! Singapore. Kuperard, London, USA. 1994.

144. Kau AK, Yang C. Values and lifestyles of Singaporeans. Singapore: NUS. 1991

145. Soh S, Frederick TL Leong. Validity of vertical and horizontal individualism and collectivism in Singapore: Relationships with values and interests. Journal of Cross - Cultural Psychology. 2002;33(1):3-15.

146. Chi WP, Ray $\mathrm{P}$, Wee V, et al. The right to protection from marital rape. Retrieved from Action for Women for Action and Research (AWARE). 2012.

147. Martin EK, Taft CT, Resick PA. A review of marital rape. Aggression and Violent Behaviour. 2007;12(3):329-347.

148. Wilkinson E. Learning to love again: 'broken families', citizenship and the state promotion of coupledom. Geoforum. 2013;49:206-213.

149. Campbell JC, Webster D, Koziol McLain J, et al. Risk factors for femicide in abusive relationships: Results from a multisite case control study. American Journal of Public Health. 2003;93(7):1089-1097.

150. Carr JL, Van Deusen KM. Risk factors for male sexual aggression on college campuses. Journal of Family Violence. 2004;19(5):279-289.

151. Markus HR, Kitayama S. Culture and the self: Implications for cognition, emotion, and motivation. Psychological Review. 1991;98(2):224-253.

152. Fiske A, Kitayama S, Markus HR, et al. The cultural matrix of socia psychology. In D. Gilbert, S. Fiske, \& G. Lindzey, The handbook of social psychology (4th ed), Vol. 2, McGraw-Hill, San Francisco, California, USA. 1998. p.915-981.

153. Bowlby J. Attachment and Loss, (Vol 2). Separation: Anxiety and Anger New York: Basic Books. 1973.

154. Chao R, Tseng V. Parenting of Asians. In: MH Bornstein (Ed), Handbook of parenting: (Vol. 4). Social conditions and applied parenting (2nd ed) Mahwah, New Jersey: Lawrence Erlbaum Associates. 2002. p. 59-93.

155. Ho, DYF. Chinese patterns of socialization: A critical review. In: Bond MH (Ed), The psychology of Chinese people Oxford University Press. Hong Kong. 1996. p.1-37.

156. Marshall WL, Marshall LE, Serran GA, et al. Rehabilitating sexual offenders: Astrength-based approach. American Psychological Association, Washington, DC, USA. 2011.

157. Ward T. Sexual offenders' cognitive distortions as implicit theories Aggression and Violent Behaviour. 2000;5(5):491-507.

158. Süssenbach P, Bohner G, Eyssel F. Schematic influences of rape myth acceptance on visual information processing: An eye-tracking approach Journal of Experimental Social Psychology. 2012;48(3):660-668.

159. Bergen RK. Wife rape: Understanding the response of survivors and service providers. Sage, Thousand Oaks, California, USA. 1996.

160. Russell DEH. Rape in marriage. Indiana University Press, Bloomington, USA. 1990

161. Bergen R. Marital rape: New research and directions. Harrisburg, PA: VA Wnet, a project of the National Resource Center on Domestic Violence Pennsylvania Coalition against Domestic Violence. 2006.

162. Lalumiere ML, Quinsey VL. The discriminability of rapists from nonsex offenders using phallometric measures: A meta-analysis. Criminal Justice and Behaviour. 1994;21(1):150-175.

163. Hall GCN, Shondrick DD, Hirschman R. The role of sexual arousal in sexually aggressive behaviour: A meta-analysis. Journal of Consulting and Clinical Psychology. 1993;61(6):1091-1095.

164. Thornhill R, Thornhill NW. The evolutionary psychology of men's coercive sexuality. Behavioural and Brain Sciences. 1992;15(2):363-375. 
165. Avery Clark C, Laws DR. Differential erection response patterns of sexual child abusers to stimuli describing activities with children. Behaviour Therapy. 1984;15(1):71-83.

166. Hall GC. Sexual arousal and arousability in a sexual offender population. Journal of Abnormal Psychology. 1989;98(2):145-149.

167. Hall GCN, Proctor WC, Nelson GM. Validity of physiological measures of pedophilic sexual arousal in a sexual offender population. Journal of Consulting and Clinical Psychology. 1988;56(1):118-122.
168. Laws DR, Holmen ML. Sexual Response Faking by pedophiles. Criminal Justice and Behaviour. 1978;5(4):343-356.

169. Quinsey VL, Steinman CM, Bergersen SG, et al. Penile circumference, skin conductance, and ranking responses of child molesters and "Normals" to sexual and nonsexual visual stimuli. Behaviour Therapy. $1975 ; 6(2): 213-219$

170. Wydra A, Marshall WL, Earls, et al. Identification of cues and control of sexual arousal by rapists. Behav Res Ther. 1983;21(5):469-476. 